\title{
Artículos
}

\section{Estructura territorial de las ciudades multiculturales y salud urbana en Oaxaca, México: un análisis bayesiano}

\section{Territorial structure of multicultural cities and urban health in Oaxaca, Mexico: A Bayesian analysis}

Andrés Enrique Miguel Velasco ${ }^{\mathrm{a}}$

Andrés Miguel Cruz

Karina Aidee Martínez García ${ }^{c}$

Luz Astrid Martínez Sánchez ${ }^{\mathrm{d}}$

Lizbeth Fabiola García Cruz

\section{Resumen}

El artículo analiza la influencia de la estructura territorial en la salud de las ciudades de Oaxaca. Plantea como hipótesis que esta influencia se manifiesta a través de la funcionalidad urbana. Utiliza

a Instituto Tecnológico de Oaxaca, División de Estudios de Posgrado e Investigación. Dirección: Ing. Víctor Bravo Ahuja 125, 68030, Oaxaca, Oax., México. Correo: andres.miguel@itoaxaca.edu.mx ORCID: https://orcid.org/0000-0003-3319-0499

${ }^{b}$ Guul (aplicación para proyectos de investigación). Oaxaca, Oax., México. Correo: anmic92@gmail.com ORCID: http://orcid.org/0000-0002-2258-2705

${ }^{\mathrm{c}}$ Instituto Tecnológico de Oaxaca, Doctorado en Ciencias en Desarrollo Regional y Tecnológico. Oaxaca, Oax., México. Correo: aidee1005@hotmail.com ORCID: http://orcid.org/0000-0002-8481-0902

${ }^{\mathrm{d}}$ Instituto Tecnológico de Oaxaca, Doctorado en Ciencias en Desarrollo Regional y Tecnológico. Oaxaca, Oax., México. Correo: luzastrid.ms@gmail.com ORCID: http://orcid.org/0000-0003-4047-9311

e Instituto Tecnológico de Oaxaca, Maestría en Ciencias en Desarrollo Regional y Tecnológico. Oaxaca, Oax., México. Correo: lizbethgarciacruz@gmail.com ORCID: http://orcid.org/0000-0003-3259-1186 
como metodología el enfoque bayesiano para el cálculo de los indicadores básicos, y el análisis de redes para la comprobación de la hipótesis. Concluye que la estructura territorial no puede considerarse un factor que genere inequidad sanitaria en el estado de salud de sus pobladores, aunque comienza a destacar en las ciudades grandes y medianas de Oaxaca que aún no consolidan su infraestructura, equipamiento y servicios.

Palabras clave: ciudades multiculturales; determinantes sociales; salud urbana; funcionalidad urbana; Oaxaca.

\begin{abstract}
The article analyzes the influence of the territorial structure on health in the cities of Oaxaca. It has as hypothesis that this influence is manifested through urban functionality. It uses as a methodology the Bayesian approach to calculate the basic indicators, and network analysis to test the hypothesis. It concludes that the territorial structure can not be considered a factor that generates health inequity in the health condition, although it starts to stand out in big and medium cities that do not consolidate their infrastructure, equipment and services in Oaxaca.
\end{abstract}

Keywords: multicultural cities; social determinants; urban health; urban functionality; Oaxaca.

\title{
1. Introducción
}

El artículo analiza si la estructura territorial de las ciudades de Oaxaca es un determinante social que genera inequidad en la salud de su población, ya que se propone que esta estructura condiciona la funcionalidad de las ciudades y, por consiguiente, la cantidad y calidad de la infraestructura y de servicios que poseen, incluidos los de salud. La interrogante al respecto es: ¿la estructura territorial también procede como un determinante social de la salud, es decir, forma parte de los factores o causas socioeconómicas condicionantes que, en el peor de los casos, afectan desfavorablemente la salud de la población de las ciudades? Específicamente se eligió Oaxaca debido a 
que este estado aún posee un ordenamiento territorial que no logra superar la marginación y el rezago social que padece la mayoría de sus localidades en el contexto nacional.

Se propone que la funcionalidad de las ciudades multiculturales, o de otro tipo, muestra el posicionamiento jerárquico y estratégico de cada urbe con respecto al conjunto al que pertenece y, por consiguiente, se vincula a la planeación estratégica, destacando sus ventajas en el territorio desde el punto de vista administrativo, político, económico, social, ambiental, cultural, religioso o militar. Por consiguiente, tal funcionalidad deriva de las fortalezas y oportunidades que poseen las ciudades -las cuales son capaces de crear círculos de atracción y crecimiento de la población, economía, cultura, etc.-, en contraposición al círculo vicioso ocasionado por sus debilidades y amenazas -que respaldan las desigualdades, las asimetrías, los conflictos sociales y el deterioro ambiental que se refleja en el territorio, específicamente en sus microrregiones de influencia.

Con base en la interrogante señalada, el objetivo del artículo es analizar si la estructura territorial, considerada como indicador de la misma funcionalidad urbana, es un determinante de la salud en las ciudades de Oaxaca. Se parte de la hipótesis de que dicha funcionalidad, para favorecer el mejoramiento de la salud de los habitantes, debe ser un determinante predominante o estratégico de la salud dentro del conjunto de los factores socioeconómicos existentes en las ciudades analizadas, como la desigualdad, la falta de mejoramiento del desarrollo, la vivienda, la educación, la gestión pública, la economía, el medio ambiente, el bienestar, la pobreza y la marginación. En concreto, se propone que entre más consolidada esté la funcionalidad de las ciudades, será un determinante social que genere menor inequidad en el estado de salud de su población residente.

El abordaje de esta relevante temática contribuye a interpretar el papel de los factores socioeconómicos en la salud de la población, particularmente en el contexto interurbano de las ciudades, y contribuye al diagnóstico de la problemática y la planeación respectiva en las ciudades mediante la sugerencia de fortalecer su infraestructura, equipamiento y servicios para favorecer a la población residente en las mismas. 


\section{Marco conceptual}

\subsection{Las ciudades multiculturales, características}

El concepto de "ciudad", del latín civitas, se define como el área urbana que presenta una alta densidad de población, conformada por habitantes que no se dedican a las actividades agrícolas. La diferencia entre las ciudades y las localidades urbanas está dada por la densidad poblacional, el estatuto legal y otros factores. En México, el Consejo Nacional de Población (Conapo) señala que lo urbano se relaciona con el concepto de ciudad, o sea, es un espacio geográfico creado y transformado por el hombre, con una alta concentración de población socialmente heterogénea, con radicación permanente, y construcciones continuas y contiguas donde se generan funciones de producción, transformación, distribución, consumo, gobierno y residencia, existiendo servicios, infraestructura y equipamiento destinados a satisfacer las necesidades sociales y a elevar las condiciones de vida de la población (Miguel, Torres, Hernández y Moncada, 2016).

En las regiones o microrregiones donde existe una bioculturalidad destacada, es factible que existan ciudades multiculturales. Una ciudad multicultural es un espacio colectivo y continuo del territorio, adaptado y construido por culturas originarias y de otro tipo para favorecer la agrupación, la convivencia, la supervivencia y el bienestar objetivo y subjetivo de la población que cobija. Es además el lugar central que favorece la acumulación de bienes tangibles e intangibles, así como la distribución y el intercambio de mercancías. Pero también es el centro territorial del control estratégico administrativo, económico, cultural y militar de los recursos naturales por parte de los grupos sociales política y económicamente dominantes, no necesariamente provenientes de los pueblos originarios. También favorece la reproducción sociocultural, en ocasiones asimétrica, de las relaciones entre los pueblos originarios y los que no lo son.

La ciudad multicultural es un concepto que contribuye a comprender el carácter histórico de la reconfiguración de la urbe contemporánea; se refiere no sólo a las propuestas para enfrentar el impacto demográfico, social y cultural de los flujos migratorios y su inclusión en el desarrollo de la ciudad, sino también a las propuestas más ins- 
titucionalizadas sobre la gestión de las diferencias culturales (Villegas, 2016).

Las ciudades multiculturales facilitan la creación de conocimiento, la innovación tecnológica y la producción artístico-cultural basada en la mezcla de conocimientos modernos con saberes tradicionales. Hoy en día, desafortunadamente, también tienden a convertirse en lugares no sustentables en el aprovechamiento de los recursos naturales y que contaminan el medio ambiente (ONU-Hábitat, 2011). En el caso de las ciudades multiculturales oaxaqueñas analizadas, existe la aspiración de que se conviertan en lugares centrales que propicien el bienestar óptimo y sustentable de sus habitantes. Se entiende que una ciudad sustentable es la que posee la capacidad de proporcionar, en forma duradera y eficiente, la energía y los recursos para cumplir con los objetivos de los subsistemas social (bienestar social), económico (productividad), ecológico (preservación ecológica) y del espacio físico urbano (calidad físico-espacial) que requerirán las generaciones presentes y futuras que habitarán la ciudad (López, 2004), generando con este proceso diversas ventajas económicas, sociales y ambientales para todos sus habitantes (Kanuri, Revi, Espey y Kuhle, 2019, p. 22).

Respecto a las ciudades analizadas en el presente artículo, resalta su característica peculiar de que se asientan en microrregiones multiculturales donde predominan los pueblos originarios, pero resultan afectadas por la problemática de las ciudades modernas; en específico, se trata de investigar la situación de la salud de sus habitantes.

\subsection{Reflexiones sobre la salud urbana}

El reporte de la Red de Conocimiento sobre Asentamientos Urbanos (KNUS, por sus siglas en inglés) de la Comisión sobre Determinantes Sociales de la Salud de la Organización Panamericana de la Salud (julio de 2007), ha subrayado la trascendencia de los efectos de la urbanización sobre la salud humana, particularmente para quienes viven en países en vías de desarrollo (OPS, 2008); tales efectos se producen por los "determinantes sociales de la salud", es decir, por los factores o causas socioeconómicas condicionantes que, como ya 
se señaló, pueden afectar negativamente la salud de la población residente en las ciudades.

El proceso de industrialización y desarrollo ha conllevado un importante crecimiento de las urbes, siempre acompañado de enfermedades ocasionadas por las condiciones insalubres debido a aguas residuales y desechos, la exposición a contaminantes, etc., o a las transmitidas por roedores y otras plagas. Los problemas medioambientales tienen un impacto directo en la salud, principalmente en la mental y en la cardiovascular, así como en otros aspectos como el estrés laboral, el pobre desarrollo durante la infancia, etc. El entorno físico modificado por la población incluye, entre otros, el aire, el agua, el ruido y las condiciones climáticas del sitio donde se encuentra la zona urbana (que son modificadas por el cambio climático, de acuerdo con el Observatorio de Salud y Medio Ambiente de Andalucía) (Osman, 2016).

El desarrollo de las ciudades también ha acrecentado las inequidades en salud a escala intra e interurbana, a pesar de que los objetivos del desarrollo del milenio y sustentables han abordado cuestiones fundamentales como la vivienda, el transporte, la economía y el medio ambiente. David Vlahov, en la primera reunión del foro Regional de Salud Urbana de la OPS, comenta que "la urbe representa una carga para la salud, una expansión de la salud y una ventaja para la salud". Al inicio pareciera que son conceptos contradictorios; sin embargo, las ciudades se encuentran en condiciones de liderar un cambio en la evolución de algunas enfermedades transmisibles y no transmisibles (OPS, 2008).

No obstante que siempre han existido enfermedades, se presenta un aparente mejoramiento en la esperanza y calidad de vida en las zonas urbanas. De acuerdo a ONU-Hábitat, el 54\% de la población mundial habita en áreas urbanas, con un envejecimiento poblacional con crecimiento anual del $3.26 \%$, y en dónde las ciudades son responsables de más del 70\% de la contaminación emitida por las emisiones mundiales de dióxido de carbono (ONU-Hábitat, 2016).

Actualmente se sabe que la urbanización ha conllevado el desarrollo de enfermedades que no se observaban con anterioridad, propiciadas por nuevos problemas como la exposición a ruidos, la alta contaminación, la restringida disponibilidad de agua y la falta de es- 
pacios abiertos; todos estos factores representan grandes retos para la salud pública actual (Osman, 2016).

La infraestructura urbana también trae consigo una enorme cantidad de desechos. Muchas ciudades no tienen un adecuado saneamiento, lo que trae consigo la contaminación del aire, suelo y agua. Los edificios, en específico, emplean grandes cantidades de energía (para calefacción y aire acondicionado, entre otros) y tienen un elevado consumo de agua; además generan una gran cantidad de residuos. Todo esto tiene efectos sobre la salud, como las enfermedades respiratorias, las alergias, las afecciones cardiovasculares, la depresión, el aislamiento, el cáncer y la obesidad (Galea y Vlahov, 2005).

Sumado a la deforestación, a la pérdida de biodiversidad y al cambio en el uso del suelo y en el consumo, la urbanización es causa de un sellado en el suelo que incrementa las inundaciones y produce islas de calor en las zonas asfaltadas, lo que aumenta la radiación infrarroja a niveles suficientes para producir golpes de calor. Todo ello merma la protección de la población frente a eventos climáticos y enfermedades vectoriales (Osman, 2016).

En México, Reyes et al. realizaron una encuesta poblacional entre 6658 familias de colonias urbanas pobres de cinco regiones geográficas de México, con objeto de evaluar las necesidades de salud de la población residente. Concluyeron que la vulnerabilidad sanitaria de las personas que viven en las áreas urbanas marginadas del país se manifiesta con trastornos y enfermedades como la desnutrición infantil, el alto riesgo reproductivo y las adicciones (Reyes et al., 2009).

Ante este proceso acelerado de urbanización, que está superando la capacidad de las autoridades locales, la Organización Mundial de la Salud plantea crear nuevas rutas que permitan combatir no sólo las enfermedades que aumentan día con día su prevalencia, como los padecimientos mentales, sino disminuir las inequidades propiciadas por los determinantes ambientales y sociales de la salud. Plantea que se deben enfocar esfuerzos en combatir la malnutrición y sobrealimentación, proporcionar agua limpia y saneamiento, transformar la movilidad urbana, mejorar la salud del hogar y garantizar la seguridad en las ciudades (OMS, 2016).

Cuando se presenta un proceso acelerado de urbanización que rebasa la capacidad de las autoridades locales, se crean nuevas co- 
munidades vulnerables, sobre todo en la periferia de las ciudades latinoamericanas; en éstas pocas veces se planifica su desarrollo o el de sus microrregiones de influencia. En dichos asentamientos informales, la transmisión de enfermedades se ve facilitada por una combinación de factores como las malas condiciones de vida, la desconexión de las infraestructuras, la escasez de servicios públicos y la inadecuada cobertura de los servicios de salud. En el caso de Oaxaca, ahí radican grupos originarios de la población, que padecen problemas específicos de desarrollo y salud.

En cambio, si el proceso de urbanización se realiza con políticas públicas que tomen en cuenta la multiculturalidad, la gobernanza y la gobernabilidad, se puede presentar el primer paso hacia la cobertura sanitaria universal en salud, con un crecimiento heterogéneo de las poblaciones urbanas, a pesar de sus diferencias sociales, culturales e inclusive económicas. De aquí la importancia de propiciar el mejoramiento de las urbes, que tarde o temprano tiende a reflejarse en el progreso de su funcionalidad.

El proceso de urbanización, al ser dinámico y complejo, requiere que el personal de salud pública trabaje de forma interdisciplinaria con distintas áreas para trascender en la salud de la población y lograr un adecuado desarrollo sustentable; asimismo, para propiciar que las poblaciones tengan herramientas y conocimientos -derivados de políticas de salud interculturales- para enfrentarse a los constantes cambios económicos, sociales, tecnológicos y ambientales que se presentan en la actualidad, así como a las diferencias que conlleva la multiculturalidad.

En algunas regiones, como las analizadas en este artículo, a la problemática señalada se aúna las vinculadas con los problemas sociales ya existentes, como la desigualdad, la falta de empleo y un ingreso digno, la marginación y la pobreza, por mencionar algunos; todos ellos son condicionantes sociales de la salud.

En este contexto se analiza el papel de la estructura territorial y la funcionalidad urbana como un indicador que manifiesta sus efectos a escala intra e interurbana, ya que la infraestructura, el equipamiento y los servicios que conlleva, convierten a la urbe en un atractor de oportunidades en el territorio: entre más consolidada esté la estructura territorial, tiende a favorecer mejores niveles de ingre- 
so, educación y salud en la población, entre otros; es decir, la funcionalidad urbana adquiere un papel estratégico.

\subsection{La estructura territorial y los determinantes socioeconómicos de la salud urbana}

La funcionalidad de las ciudades está ligada a su estructura territorial, pues esta última representa el soporte básico de un país "donde interaccionan los centros, responsables de la organización regional, a través de ejes articuladores del espacio" (Propin y Sánchez-Crispín, 2001). Este análisis permite conocer la distribución de los asentamientos,

[...] las interrelaciones espaciales, los grados de especialización y jerarquización, las potencialidades y deficiencias existentes, la delimitación de las unidades internas, y señalar los elementos esenciales para determinar las líneas fundamentales de la política territorial [Gómez, 1999, p. 363].

En este proceso, el análisis funcional del sistema territorial tiene por objeto "determinar la contribución de los elementos a la funcionalidad del conjunto, y la posición que ocupan en el mismo" (Gómez, 1999, p. 363).

En el presente artículo se analiza la funcionalidad urbana con base en la visión de la planeación estratégica, debido a que ésta facilita el examen de las fortalezas y debilidades existentes en el territorio de las ciudades, las cuales son los factores o recursos endógenos de las urbes sobre los que se tiene algún grado de control. Esta perspectiva también permite analizar la situación exógena que rodea a la ciudad y la problemática de su contexto, con objeto de visualizar las oportunidades y amenazas existentes, y de las cuales se tiene poco o ningún control directo (Fernández, 2006).

Se propone que las fortalezas de las urbes se consolidan a través de sus logros en materia de educación, vivienda, etc., y se materializan en infraestructura, equipamiento y servicios tangibles (agua potable, electricidad, drenaje, etc., así como los específicos 
en materia de salud). Ello, aunado a la cantidad de población que ahí radica, al crecimiento económico, las inversiones, la creación de empleos, el desarrollo turístico o los apoyos en programas públicos, entre otros, genera ventajas para las ciudades y para su gestión pública exitosa.

En cuanto a las debilidades urbanas, se supone que son producto de las desigualdades y la concentración anárquica de la infraestructura, en unión con amenazas como el cambio climático o los fenómenos naturales, las enfermedades, así como los conflictos sociales y las crisis económicas que se desarrollan en su territorio. Todo ello se refleja en aspectos externos, tangibles e intangibles, que debilitan su mejoramiento, generando desventajas y círculos viciosos que debilitan la calidad de vida de su población. Esto se inserta en un contexto cultural complejo en el que los grupos originarios, a escala intra e interurbana, se enfrentan a la exclusión y luchan por lograr el reconocimiento de sus aportaciones a las ciudades en las que habitan.

La propuesta concreta es que las ciudades cuyas fortalezas y oportunidades son superiores a sus debilidades y amenazas, tienden a mejorar su jerarquía en la red de localidades, su competitividad, así como el nivel de vida y la salud de su población, llegando a consolidar su funcionalidad, la cual se refleja en más inversiones en empresas privadas y públicas, atrayendo más infraestructura y servicios, incluidos los del sector salud.

Por el contrario, las ciudades cuyas fortalezas y oportunidades no alcanzan a superar sus debilidades y amenazas, quedan rezagadas en su desarrollo, pierden competitividad, frenan su desarrollo sustentable y tienden a convertirse en la periferia de las metrópolis con mayor jerarquía o funcionalidad, lo que afecta la calidad de vida de su población, incluida la salud urbana correspondiente.

Se espera que entre mayor sea la jerarquía y funcionalidad de las ciudades, la salud de su población residente resultará más beneficiada. 


\section{Procedimiento metodológico}

\subsection{Indicadores de la base de datos}

El análisis de la información se fundamenta en un enfoque cuantitativo que considera los valores estandarizados de los indicadores para obtener índices de cada componente (IC) y permitir su comparación. Los indicadores obtenidos se muestran en el Cuadro 1.

Para la valoración de la funcionalidad urbana y sus componentes, se seleccionaron indicadores de población de los censos y conteos de la población del Instituto Nacional de Estadística y Geografía (INEGI, 2000; 2005; 2010; 2015); del Centro de Información Estadística y Documental para el Desarrollo (CIEDD, 2014); y del índice de desarrollo humano y el producto interno bruto del Programa de las Naciones Unidas para el Desarrollo (PNUD, 2015). Los indicadores ambientales de emisiones y el consumo de agua por habitante (cantidad de agua que consume una persona para beber, limpieza, preparación de alimentos y otros usos domésticos), así como los de la generación de residuos sólidos para los municipios más urbanizados del estado de Oaxaca, se obtuvieron de la Secretaría de Medio Ambiente y Recursos Naturales (Semarnat, 2015). Como indicador económico se consideró la actividad turística, debido a su importancia e incidencia en todas las ciudades analizadas; se contó con una base de datos de la Secretaría de Turismo (Sectur, 2013) que cubre todos los indicadores del conjunto de los datos utilizados en el análisis, considerando el total de turistas, los recursos para el fomento turístico, el personal y la profesionalización del sector hotelero, la infraestructura y la accesibilidad, así como los servicios complementarios al turismo, Los datos de los conflictos en las ciudades fueron obtenidos de la Secretaría de Vialidad y Transporte (Sevitra, 2017) del estado de Oaxaca.

Con respecto a los indicadores de salud, se tomaron en cuenta los siguientes: del INEGI, el índice de envejecimiento (2013a), los indicadores de la serie censal (1990, 2000 y 2010) y el índice de pobreza inercial (2013a). De la Secretaría de Salud (Dirección General de Información en Salud, DGIS, 2000-2016), cubos dinámicos, el índice de mortalidad infantil (compuesto por el número de defunciones de 
menores de un año divididos por cada mil nacimientos); el índice de desnutrición (calculado como el porcentaje de defunciones ocasionadas por desnutrición calórico-proteica, anemia, bajo peso al nacimiento y prematurez, dividido por el total de defunciones en cada localidad); el índice de obesidad (porcentaje de muertes ocasionadas por diabetes mellitus, enfermedades hipertensivas, enfermedades cerebrovasculares y enfermedades isquémicas del corazón, dividido por el total de defunciones en cada localidad); la tasa de mortalidad general (considerando los condicionantes derivados de la obesidad, en el que el riesgo de desarrollar enfermedad cerebrovascular y cardiopatía isquémica es dos veces mayor, y el de padecer diabetes mellitus es 93 veces mayor, en comparación con la ausencia de obesidad) (Guerra, 2009; Rodríguez, 2003).

\subsection{La prueba de hipótesis}

Los indicadores descritos se transforman en probabilidades de ocurrencia mediante el teorema de Bayes, el cual es de gran importancia en las ciencias de la salud, y es especialmente utilizado en el cálculo de probabilidades diagnósticas (Mercado y Robles, 2016). Dicho teorema parte de una situación en la que es posible conocer las probabilidades de que ocurra una serie de sucesos, $A i$, conociendo que ha acontecido el suceso Bi (Salinas, 2019).

En el análisis de la problemática de las ciudades, el teorema de Bayes es de utilidad cuando se requiere analizar el contexto en el que se desenvuelven las variables analizadas. A través del mismo, se visualiza la probabilidad de ocurrencia de estas variables con relación a su entorno. Aplicado al campo de la salud urbana, el teorema de Bayes contribuye a destacar la importancia de mejorar la detección de los determinantes predominantes de la salud, entre otros aspectos, reduciendo la valoración de aquellos con baja probabilidad de ocurrencia, lo cual puede incidir en la realización de mejores diagnósticos de salud en las ciudades ya que, según el nivel de probabilidad obtenido a través del teorema, los recursos destinados, a partir de la generalización de las decisiones, pueden emplearse potencialmente en otros proyectos de mayor trascendencia. Es decir, sugiere tomar 
decisiones encaminadas al uso más eficiente de los recursos con base en los determinantes con mayor probabilidad de ocurrencia en el territorio o las ciudades.

\section{Cuadro 1}

Indicadores de la funcionalidad urbana y la salud urbana

\begin{tabular}{|c|c|c|}
\hline \multicolumn{2}{|c|}{ Funcionalidad urbana } & \multirow{2}{*}{$\begin{array}{l}\text { Modelo de análisis } \\
\text { FEST }= \\
\text { (FORT+OPOR)- } \\
\text { (DEBI+AMEN) }\end{array}$} \\
\hline $\begin{array}{l}\text { Fortalezas } \\
\text { (FORT) }\end{array}$ & $\begin{array}{l}\text { EDUC: educación } \\
\text { POB: población } \\
\text { VIV: vivienda } \\
\text { DSUS: desarrollo sustentable } \\
\text { AMB: medio ambiente }\end{array}$ & \\
\hline $\begin{array}{l}\text { Oportunidades } \\
\text { (OPOR) }\end{array}$ & $\begin{array}{l}\text { TUR: turismo } \\
\text { GEST: gestión pública }\end{array}$ & $I_{C}=\frac{V_{R}-V_{\text {min }}}{V_{\text {máx }}-V_{\text {min }}}$ \\
\hline $\begin{array}{l}\text { Debilidades } \\
\text { (DEBI) }\end{array}$ & $\begin{array}{l}\text { DESG: desigualdades sociales } \\
\text { CONC: concentración territorial } \\
\text { extrema } \\
\text { POBRE: pobreza } \\
\text { MARG: marginación }\end{array}$ & $\begin{array}{l}I_{C}: \text { índice de componente } \\
V_{R}: \text { valor real } \\
V_{\text {máx }}: \text { valor máximo } \\
V_{\text {mín }}: \text { valor mínimo }\end{array}$ \\
\hline $\begin{array}{l}\text { Amenazas } \\
\text { (AMEN) }\end{array}$ & $\begin{array}{l}\text { CC: cambio climático } \\
\text { CONF: conflictos sociales }\end{array}$ & \\
\hline \multicolumn{3}{|c|}{ Salud urbana } \\
\hline $\begin{array}{l}\text { Envejecimiento } \\
\text { (ENVEJ) }\end{array}$ & ENVEJ: índice de envejecimiento & $\begin{array}{l}\text { donde: } \\
\mathrm{I}_{\mathrm{T}}=\sum \mathrm{I}_{\mathrm{C}} \\
\text { Probabilidad de } \\
\mathrm{I}_{\mathrm{C}}=\mathrm{I}_{\mathrm{C}} / \mathrm{I}_{\mathrm{T}}\end{array}$ \\
\hline $\begin{array}{l}\text { Mortalidad infantil } \\
\text { (MORTINF) }\end{array}$ & $\begin{array}{l}\text { MORTINF: tasa de mortalidad } \\
\text { infantil }\end{array}$ & \\
\hline $\begin{array}{l}\text { Desnutrición } \\
\text { (DESNUT) }\end{array}$ & DESNUT: índice de desnutrición & \\
\hline $\begin{array}{l}\text { Obesidad } \\
\text { (OBES) }\end{array}$ & OBES: índice de obesidad & \\
\hline $\begin{array}{l}\text { Mortalidad } \\
\text { (MORT) }\end{array}$ & MORT: mortalidad & \\
\hline $\begin{array}{l}\text { Pobreza crónica } \\
\text { (POB) }\end{array}$ & POB: índice de pobreza crónica & \\
\hline
\end{tabular}

Fuente: Elaboración propia con base en INEGI $(2000 ; 2005 ; 2010 ; 2015)$, PNUD (2015), Sectur (2013), Semarnat (2015) y Sevitra (2017). 
En el caso específico de este artículo, y con base en la probabilidad de los índices del Cuadro 1, este teorema se utiliza para determinar la probabilidad de ocurrencia de los determinantes sociales de la salud $\left(\mathrm{I}_{\mathrm{D} 1}, \mathrm{I}_{\mathrm{D} 2} \ldots \mathrm{I}_{\mathrm{D} 15}\right)$, según los indicadores $\left(\mathrm{I}_{\mathrm{A}}, \mathrm{I}_{\mathrm{B}}, \mathrm{I}_{\mathrm{C}}, \ldots, \mathrm{I}_{\mathrm{N}}\right)$, en las ciudades analizadas. El modelo del cálculo respectivo es:

$$
\begin{aligned}
\mathrm{P}\left(\mathrm{I}_{\mathrm{Di}}\right)_{\mathrm{j}}=\mathrm{P}\left(\mathrm{I}_{\mathrm{A}}\right) \times \mathrm{P}\left(\mathrm{I}_{\mathrm{Di}} / \mathrm{I}_{\mathrm{A}}\right) /\left[\mathrm{P}\left(\mathrm{I}_{\mathrm{A}}\right) \times \mathrm{P}\left(\mathrm{I}_{\mathrm{Di}} / \mathrm{I}_{\mathrm{A}}\right)\right]+\left[\mathrm{P}\left(\mathrm{I}_{\mathrm{B}}\right) \times \mathrm{P}\left(\mathrm{I}_{\mathrm{Di}} / \mathrm{I}_{\mathrm{B}}\right)\right] \\
+\ldots+\left[\mathrm{P}\left(\mathrm{I}_{\mathrm{N}}\right) \times \mathrm{P}\left(\mathrm{I}_{\mathrm{Di}} / \mathrm{I}_{\mathrm{N}}\right)\right]
\end{aligned}
$$

Se seleccionaron como determinantes predominantes los de mayor probabilidad de ocurrencia en las ciudades analizadas.

La hipótesis también se valora considerando que los determinantes de salud adquieren predominio en el conjunto a través de las redes que las ciudades conforman en su interacción (Hernández, 2016). En este caso, las redes territoriales describen el comportamiento económico, social o de salud de las ciudades. Se convierten en una herramienta teórico-metodológica para el análisis de la asociación y medida de las relaciones y flujos entre las personas, las mercancías, las organizaciones o los determinantes de la salud de las urbes, ya que los nodos en las redes muestran los enlaces establecidos entre los elementos seleccionados (Salingaros, 2005; Serrano 2000).

La ordenación de los elementos determina a su vez la jerarquía de éstos en el conjunto y, por consiguiente, su capacidad para convertirse en elementos dinámicos del mismo. En este caso, los elementos seleccionados se tornan en nodos de la red, $G=(V, E)$, donde $\mathrm{V}$ es su conjunto de vértices (v) y E su conjunto de aristas.

Estas redes se caracterizan por las propiedades de centralización, que es la cualidad de que exista algún nodo dominante en la red. La centralidad se refiere a la posición de los lugares centrales en la red, en la cual la centralidad de grado $(\mathrm{C})$ se define como $\mathrm{C}(\mathrm{v})=$ grado (v), siendo la centralidad de la red; y la densidad de las interacciones refleja el tejido de interacciones existente entre los determinantes de salud de las ciudades.

Se espera que la funcionalidad posea un mayor nivel de centralidad dentro del conjunto de los factores socioeconómicos más reconocidos, como la desigualdad, la marginación, etc., con respecto a los determinantes de salud, en el sentido de que, en la red, una mayor 
centralidad del elemento que destaque tiende a reflejar un mejor apoyo de la ciudad a la salud de sus habitantes.

Para la construcción de la red y sus indicadores, se utilizó el programa Ucinet Tecnologías Analíticas versión 6.6 para Windows, cuya versatilidad facilita la obtención de las redes, tanto en el análisis territorial, como en el económico-social o de salud.

\section{Resultados}

\subsection{Contexto territorial de la salud en Oaxaca}

El estado de Oaxaca está situado en la porción meridional de la República Mexicana. Limita al norte y noreste con Veracruz y Puebla, al este con Chiapas, al sur con el océano Pacifico y al oeste con Guerrero (Mapa 1). Se creó por decreto del Congreso de la Unión el 3 de febrero de 1824. Políticamente está dividido en 30 distritos y 570 municipios (24\% de los municipios del país). Tiene una extensión de $91783 \mathrm{~km}^{2}$, lo que representa el $4.8 \%$ de la superficie nacional, que lo coloca como el quinto estado más grande de la República (INEGI, 2013; Ordóñez, 2000). En 2012, Oaxaca contaba con 3040962 habitantes, de los cuales el $39.56 \%$ hablaba alguna lengua originaria (mayores a tres años de edad). En esta entidad se registran aproximadamente 66 lenguas originarias, siendo las cinco principales: zapoteco $(30.9 \%)$, mixteco $(21.9 \%)$, mazateco $(14.6 \%)$, mixe $(9.8 \%)$ y chinanteco (9.1\%) (INEGI, 2013; Ordóñez, 2000).

En México, la población originaria conforma el grupo de los pueblos que, dada su cultura, historia y lengua, dan sentido de pertenencia e identidad al país. Sin embargo, son grupos en los que prevalece la pobreza y la marginación; se encuentran en un estado de vulnerabilidad que propicia que adquieran enfermedades y ello conlleva un aumento de su mortalidad (INEGI, 2016; CDI, 2006). El 96\% de los grupos originarios vive en áreas rurales remotas en México, con escasos caminos, transporte público deficiente, malas condiciones de vivienda, bajo nivel educativo, en comunidades con condiciones de pobreza extrema y alta marginación (Torres, 2003), lo que influye en sus niveles de salud. Las quince ciudades y las dos zonas metropolitanas de Oaxaca 


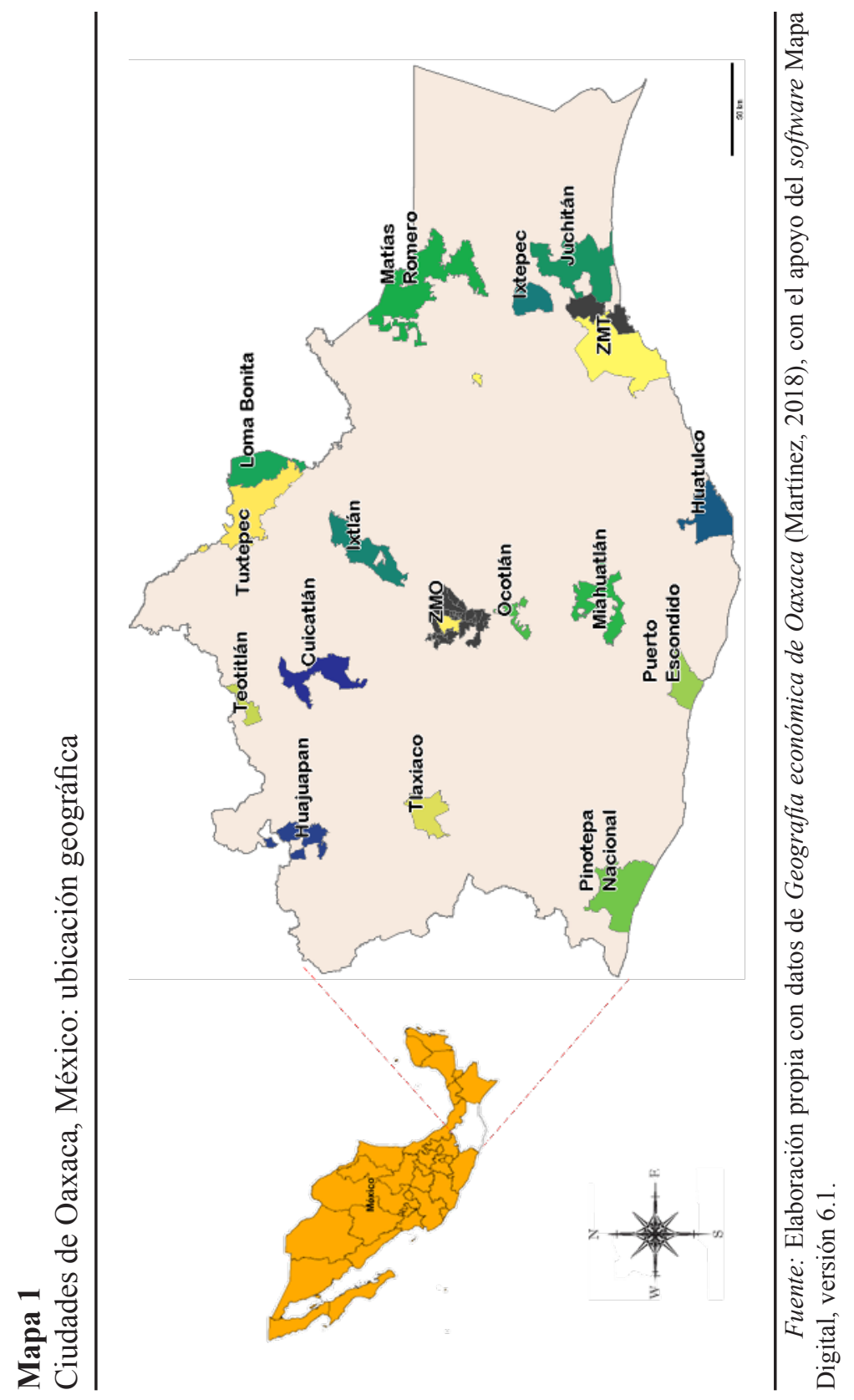


analizadas en este artículo poseen rasgos de culturas tradicionales, o bien, influyen en microrregiones conformadas por asentamientos de pueblos originarios (Cuadro 2).

\section{Cuadro 2}

Ciudades de Oaxaca, México

\begin{tabular}{|c|c|c|c|c|}
\hline \multirow[b]{2}{*}{ Ciudad* } & Abrev. & Pob. & $\begin{array}{c}\% \text { pob. } \\
\text { originaria }\end{array}$ & Región Oaxaca** \\
\hline & \multicolumn{4}{|c|}{ Pequeña } \\
\hline Ixtlán & IXN & 8268 & 61.88 & Sierra Norte $(76.3 \%)$ \\
\hline Cuicatlán & CUI & 9945 & 13.82 & Cañada $(71.5 \%)$ \\
\hline Teotitlán & TEO & 9876 & 17.83 & Cañada $(71.5 \%)$ \\
\hline Ixtepec & IXP & 22675 & 21.05 & Istmo $(31.5 \%)$ \\
\hline Loma Bonita & LOB & 40877 & 2.56 & Papaloapan (38\%) \\
\hline Matías Romero & MAR & 40709 & 12.00 & Istmo $(31.5 \%)$ \\
\hline Miahuatlán & MIA & 32555 & 9.56 & Sierra Sur $(33.6 \%)$ \\
\hline Ocotlán & $\mathrm{OCO}$ & 18183 & 3.65 & Valles Centrales (16.4\%) \\
\hline Puerto Escondido & PES & 32471 & 3.41 & Costa $(31 \%)$ \\
\hline Tlaxiaco & TLX & 29026 & 25.87 & Mixteca (36.1\%) \\
\hline Huatulco & $\mathrm{CRU}$ & 28327 & 4.25 & Costa $(31 \%)$ \\
\hline Pinotepa & PIN & 44193 & 19.04 & Costa $(31 \%)$ \\
\hline \multicolumn{5}{|c|}{ Mediana } \\
\hline Huajuapan & HJP & 53219 & 7.88 & Mixteca (36.1\%) \\
\hline Juchitán & JUC & 78512 & 57.49 & Istmo $(31.5 \%)$ \\
\hline \multicolumn{5}{|c|}{ Grande } \\
\hline Tuxtepec & TUX & 133913 & 13.42 & Papaloapan (38\%) \\
\hline \multicolumn{5}{|c|}{ Zona metropolitana } \\
\hline ZMT & ZMT & 145567 & 12.75 & Istmo $(31.5 \%)$ \\
\hline $\mathrm{ZMO}$ & $\mathrm{ZMO}$ & 501283 & 8.33 & Valles Centrales $(16.4 \%)$ \\
\hline
\end{tabular}

* Se apuntan los nombres cotidianos de las ciudades analizadas. ZMT: Zona Metropolitana de Tehuantepec. ZMO: Zona Metropolitana de Oaxaca.

** Porcentaje regional de población originaria.

Fuente: Elaboración propia con base al Sistema de Información Municipal (CIEDD, 2014).

Durante los últimos cien años, la población de Oaxaca se multiplicó casi 3.7 veces. Está distribuida en $77 \%$ en áreas urbanas y 23\% en rurales, y la entidad tienen un promedio de 41 habitantes por $\mathrm{km}^{2}$ 
(INEGI, 2010a). La ciudad de mayor tamaño poblacional, la Zona Metropolitana de Oaxaca (ZMO), cuenta con una superficie total de $602.7 \mathrm{~km}^{2}$ y una población de 659234 habs., conteniendo al 16.62\% de la población estatal (INEGI, 2015). Ixtlán es la ciudad que posee el mayor porcentaje de población originaria (61.88\%), y Loma Bonita la menor proporción (2.56\%). La ZMO, la de mayor población, posee el $8.33 \%$ de habitantes originarios. El promedio de la población originaria de las ciudades es de $17.34 \%$, y por región en la cual se asientan las ciudades, el porcentaje promedio es de $42 \%$, lo que proporciona características multiculturales exclusivas a las ciudades de Oaxaca.

\subsection{La funcionalidad de las ciudades de Oaxaca}

Con respecto a la funcionalidad (FEST) de las ciudades, las fortalezas, oportunidades, debilidades y amenazas (FODA) muestran que el valor promedio de las debilidades (DEBI, 0.41) en las ciudades de Oaxaca corresponde a un nivel medio; sin embargo, se encuentra por encima de las demás variables y se ve influenciada principalmente por las desigualdades sociales (DESG, 0.60), la cual se posiciona con el valor más alto. Dentro de las fortalezas (FORT, 0.22), el desarrollo sustentable (DSUS, 0.54) tiene el mayor aporte. En las oportunidades (OPOR, 024), el factor que tiene mayor influencia es representado por la actividad del turismo (TUR, 0.34), a pesar de que ostenta un valor bajo. Por último, las amenazas (AMEN, 0.13) se sustentan por el factor del cambio climático $(\mathrm{CC}, 0.23)$. Resulta en su conjunto la funcionalidad estratégica (FEST, -0.07) con el menor valor, como se muestra en la Gráfica 1.

Factores como las desigualdades, el desarrollo sustentable, la gestión y la vivienda, impactan particularmente en la ZMO, Puerto Escondido y la Zona Metropolitana de Tehuantepec (ZMT). La ZMO destaca con fortalezas y oportunidades consolidadas. En el rubro de debilidades, todas las ciudades se ubican en un nivel medio. Las urbes poseen un nivel bajo de amenazas, sólo Cuicatlán posee un rango muy bajo en este rubro.

En general, el promedio de la funcionalidad del conjunto de las ciudades de Oaxaca (-0.07) indica que las fortalezas y oportunidades 
no superan sus debilidades y amenazas. Las urbes en transición hacia una funcionalidad positiva son: Juchitán, ZMT, Ixtlán y Huatulco. El resto se encuentra con valores negativos de funcionalidad. Las ciudades que ya poseen valores positivos en su funcionalidad son: ZMO, Puerto Escondido, Tuxtepec y Pinotepa, las cuales son las que ofrecen las mayores oportunidades de desarrollo en la entidad.

\section{Gráfica 1}

Ciudades de Oaxaca: valor de los factores estratégicos

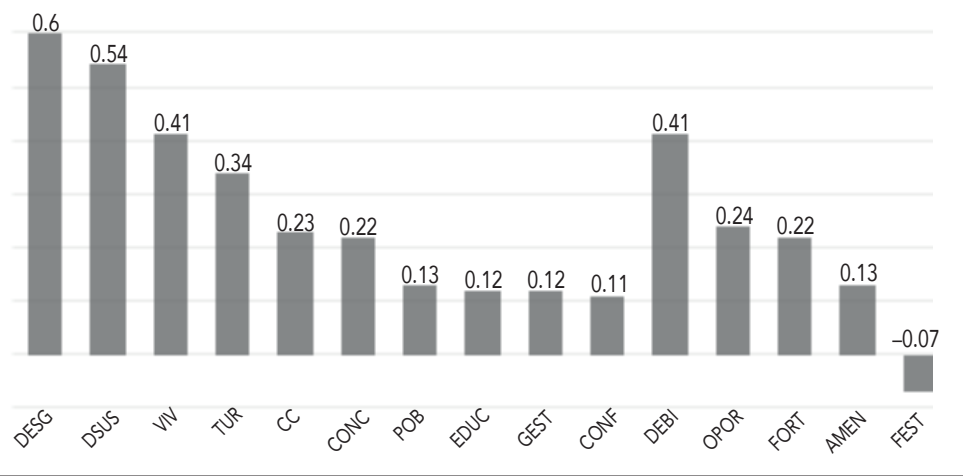

Nota: DESG: desigualdad; DSUS: desarrollo; VIV: vivienda; TUR: turismo; CC: cambio climático; CONC: concentración territorial extrema; POB: población; EDUC: educación; GEST: gestión pública; CONF: conflictos sociales; DEBI: debilidades; OPOR: oportunidades; FORT: fortalezas; AMEN: amenazas; FEST: funcionalidad.

Fuente: Elaboración propia con datos de INEGI (2000; 2005; 2010).

La estructura de la red de ciudades muestra que la funcionalidad de las urbes no coincide con su centralidad, pues las ciudades que destacan por su centralidad son: Juchitán, Tlaxiaco, Huajuapan y la ZMO; ésta última mantiene un fuerte nexo con Ocotlán (en color oscuro en la Gráfica 2). El resto de las ciudades ocupa un lugar periférico en la red por su baja centralidad, lo que indica que poseen déficits de infraestructura, equipamiento y servicios, y confirma el bajo nivel de funcionalidad de la mayoría de las ciudades de Oaxaca. 


\section{Gráfica 2}

Red de la funcionalidad urbana de las ciudades de Oaxaca

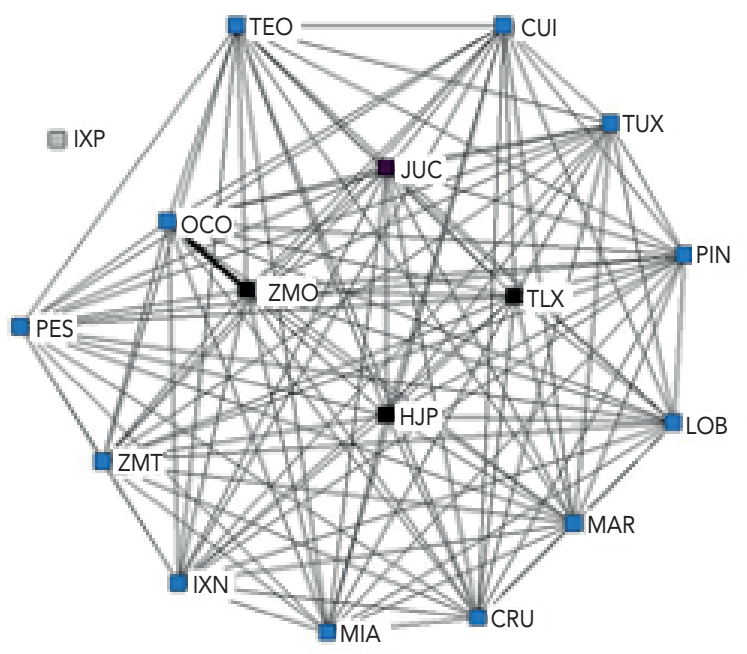

Nota: IXN: Ixtlán; CUI: Cuicatlán; TEO: Teotitlán; IXP: Ixtepec; LOB: Loma Bonita; MAR: Matías Romero; MIA: Miahuatlán; OCO: Ocotlán; PES: Puerto Escondido; TLX: Tlaxiaco; CRU: Huatulco; PIN: Pinotepa Nacional; HJP: Huajuapan; JUC: Juchitán; TUX: Tuxtepec; ZMT: Zona Metropolitana de Tehuantepec; ZMO: Zona Metropolitana de Oaxaca.

Fuente: Elaborado con datos de los Cuadros 4 y 5 , mediante el programa UCINET, 6.6.

\subsection{El contexto de la salud en Oaxaca y los pueblos originarios}

Oaxaca es un estado con barreras geográficas que limitan el acceso a los servicios y la infraestructura, lo que contribuye a incrementar las desigualdades en materia de salud (Torres et al., 2003). En el país, según la Encuesta Intercensal 2015, quince de cada cien personas hablantes de lenguas originarias no están afiliadas a los servicios de salud; los más desprotegidos son los varones (57.7\% no cuentan con dichos servicios, contra $45.3 \%$ en las mujeres). Además, hay que considerar que la afiliación por sí misma no garantiza el acceso 
a los servicios de salud, ya que existen otras barreras económicas, ambientales, culturales y administrativas. Del total de la población hablante de lengua originaria afiliada a una institución que presta servicios de salud, casi la totalidad (98.8\%) está inscrita en alguna del sector público, principalmente al Seguro Popular (72.6\%), mientras que el $0.5 \%$ cuenta con los servicios de salud de alguna institución privada (INEGI, 2016).

Una referencia de la problemática de la salud en Oaxaca se observa en los 22360 casos de defunción reportados por el INEGI (2016), de los cuales $46.2 \%$ son mujeres y $53.8 \%$ son hombres, con una media de 66.55 años de edad.

De los casos estudiados, 6203 personas (33.3\%) no tenían ninguna seguridad social. Los que sí gozaban de este derecho contaban con Seguro Popular, 39.6\%; Instituto Mexicano del Seguro Social (IMSS), 12.6\%; Instituto de Seguridad y Servicios Sociales de los Trabajadores del Estado (ISSSTE), 7.2\%; IMSS Oportunidades, 4.4\%; Petróleos Mexicanos (Pemex), 0.9\%; Secretaria de la Defensa Nacional (Sedena), 1.0\%; otros, 0.5\%; y Secretaría de Marina Armada (Semar), 0.4\%.

En cuanto a la condición de atención médica, 75.5\% (15691 personas) recibió atención médica y $24.5 \%$ no la tuvo. Respecto a las defunciones, las cinco primeras causas (lista mexicana) fueron: diabetes mellitus, $12.9 \%$ (2883 personas); infarto agudo del miocardio, 9.1\%; otras enfermedades del hígado, 3.7\%; homicidios, 3.3\%; y otras desnutriciones proteinocalóricas, $2.9 \%$.

En la muestra descrita, 9032 personas manifestaron ser hablantes de alguna lengua originaria (40.39\% del total); 49\% eran mujeres y $51 \%$, hombres, con una media de 70.91 años de edad. Las ocupaciones que se presentaron con mayor frecuencia fueron: trabajadores en actividades agrícolas, ganaderas, forestales, caza y pesca (51.6\%); trabajadores artesanales $(3.5 \%)$; y comerciantes, empleados en ventas y agentes de ventas (1.6\%).

En cuanto al nivel de escolaridad en la muestra total con condición de lengua originaria, se registró una mayor frecuencia en los casos sin escolaridad (54.8\%), seguidos por quienes tenían la primaria incompleta $(39.1 \%)$, secundaria completa $(2.6 \%)$, profesional $(1.6 \%)$, secundaria incompleta $(1.2 \%)$, bachillerato completo 
$(0.8 \%)$ y bachillerato incompleto $(0.4 \%)$. Sólo existían tres casos con posgrado. Con respecto al sexo, según el nivel de escolaridad con condición de lengua originaria, el grupo femenino tuvo un mayor porcentaje sin escolaridad (69.40\%), comparado con el masculino (40.90\%); con primaria incompleta se registró 38.10\% en los hombres, mientras que $21.8 \%$ en las mujeres; con nivel profesional hubo $2.60 \%$ entre los hombres, y en las mujeres sólo 0.50\% (Cuadro 3).

\section{Cuadro 3}

Nivel de escolaridad de acuerdo

al sexo y condición de lengua originaria

\begin{tabular}{|c|c|c|c|c|}
\hline \multirow[b]{3}{*}{ Escolaridad } & \multicolumn{4}{|c|}{ Sexo } \\
\hline & \multicolumn{2}{|c|}{ Masculino } & \multicolumn{2}{|c|}{ Femenino } \\
\hline & Frecuencia & Porcentaje & Frecuencia & Porcentaje \\
\hline Sin escolaridad & 1835 & 40.9 & 2987 & 69.4 \\
\hline Preescolar & 9 & 0.2 & 3 & 0.1 \\
\hline $\begin{array}{l}\text { Primaria } \\
\text { incompleta }\end{array}$ & 1707 & 38.1 & 939 & 21.8 \\
\hline $\begin{array}{l}\text { Primaria } \\
\text { completa }\end{array}$ & 487 & 10.9 & 243 & 5.6 \\
\hline $\begin{array}{l}\text { Secundaria } \\
\text { incompleta }\end{array}$ & 70 & 1.6 & 36 & 0.8 \\
\hline $\begin{array}{l}\text { Secundaria } \\
\text { completa }\end{array}$ & 182 & 4.1 & 50 & 1.2 \\
\hline $\begin{array}{l}\text { Bachillerato } \\
\text { incompleto }\end{array}$ & 23 & 0.5 & 9 & 0.2 \\
\hline $\begin{array}{l}\text { Bachillerato } \\
\text { completo }\end{array}$ & 55 & 1.2 & 16 & 0.4 \\
\hline Profesional & 116 & 2.6 & 22 & 0.5 \\
\hline Posgrado & 1 & 0.0 & 2 & 0.0 \\
\hline Total & 4485 & 100.0 & 4307 & 100.0 \\
\hline
\end{tabular}

Fuente: Elaboración propia con base en los tabulados de la Encuesta Intercensal (INEGI, 2015).

En cuanto a la seguridad social en los pobladores con condición de lengua originaria, se presentaron afiliados en orden descendente al: Seguro Popular, 41.5\%; ninguna afiliación, 29.35\%; IMSS Opor- 
tunidades, 7\%; IMSS, 5.7\%; ISSSTE, 4.7\%; Sedena, 1\%; Pemex, $0.7 \%$; otra, $0.6 \%$; y SEMAR, $0.4 \%$ (Gráfica 3).

\section{Gráfica 3}

Frecuencias de derechohabiencia

con condición de lengua originaria

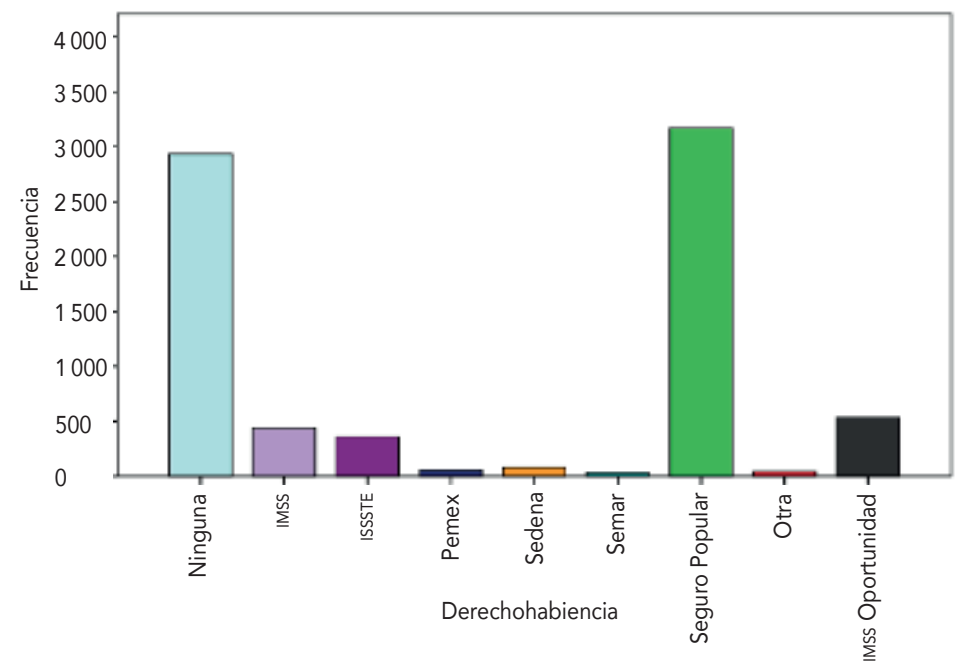

Fuente: Elaboración propia con base en la Encuesta Intercensal (INEGI, 2015).

Las principales causas de defunción en los pobladores con condición de lengua originaria son: diabetes mellitus, $13.3 \%$ (102 personas); infarto agudo al miocardio, $10.4 \%$; otras desnutriciones proteinocalóricas, 3.8\%; otras enfermedades del hígado, 3.8\%; y senilidad, 3.5\%.

En los casos considerados, al comparar los grupos originarios con el resto de la población, se observa que la edad media de defunción para Oaxaca es de 67 años, teniendo un mayor promedio el grupo de lengua originaria, con una media de 71; esto no necesariamente un indicador de una mejor esperanza de vida para el grupo de lengua originaria. En Oaxaca se registra un mayor porcentaje de mortalidad en el sexo masculino (53.8\%), contra el del sexo femenino (46.2\%); mientras que en el grupo de lengua originaria hay un mayor porcen- 
taje de muertes entre las mujeres (51\%), en comparación con el de los hombres $(49 \%)$.

Con respecto a la ocupación, la principal actividad en Oaxaca y en los grupos originarios es trabajador en actividades agrícolas, ganaderas, forestales, caza y pesca (32.9 y 50\% respectivamente), siendo más marcada esta actividad en el grupo con lengua originaria. En ambos grupos, un porcentaje considerable no labora (52.1\% para Oaxaca y $51.6 \%$ para los hablantes de lengua originaria). La ocupación se analizó por edad y sexo, siendo mayor la edad de defunción en los trabajadores en actividades agrícolas, ganaderas, forestales, caza y pesca, en comparación con el 50\% del resto de las ocupaciones. Resalta que el $77 \%$ del sexo masculino fallece en esta ocupación.

En cuanto a la escolaridad de los fallecidos, en ambos casos se tiene un predominio de "sin escolaridad", con 40.5\% para Oaxaca, y $54.8 \%$ en el grupo de lengua originaria. Al analizar la escolaridad con la edad en el grupo de lengua originaria, se presentó una mayor edad de mortalidad para el grupo sin escolaridad, comparado con la edad máxima de posgrados. De acuerdo al sexo, para el máximo grado académico (posgrado) se tiene un porcentaje muy bajo para ambos sexos, comparado con el grupo que no tiene educación, con $69.50 \%$ para las mujeres y $40.90 \%$ para los hombres.

Respecto a la seguridad social o afiliación, prevalece "sin ninguna seguridad social", con $33.3 \%$ en Oaxaca, comparado con los hablantes de lengua originaria, con $29.35 \%$. La afiliación principal en el grupo originario es el Seguro Popular (41.5\%), comparado con Oaxaca $(39.6 \%)$.

Las principales causas de defunción, tanto en la población total de Oaxaca como en el grupo de lengua originaria, son las enfermedades no transmisibles, crónico-degenerativas, evitables como diabetes mellitus, e infarto agudo al miocardio. Existe una diferencia en el tercer lugar: en Oaxaca la causa es "otras enfermedades del hígado", mientras que en el grupo de lengua originaria dicho lugar lo ocupa "otras desnutriciones proteinocalóricas".

En cuanto a la condición de atención médica, se recibió $75.5 \%$ en Oaxaca y $69.9 \%$ en los grupos originarios, siendo el área rural donde existe una mayor cantidad de fallecidos para el grupo de lengua originaria $(63.5 \%)$. 
Las localidades de residencia habitual con más defunciones para el grupo de lengua originaria, son: Oaxaca, 7.5\%; Juchitán, 4.3\%; e Ixtepec, $1.3 \%$. Hay un tamaño de localidad de residencia similar en ambos grupos, siendo el rango donde más defunciones se presentaron el de 1 a 999 habitantes: $34.2 \%$ en Oaxaca y $41.1 \%$ en el grupo de lengua originaria.

\subsection{Comportamiento de la salud interurbana en Oaxaca}

En este apartado se analiza el papel de los determinantes sociales de la salud, con la finalidad de analizar su relación con la funcionalidad de las ciudades. Tomando como referencia la problemática nacional en la cual las enfermedades derivadas de la actividad urbana (como la falta de ejercicio y la sobrealimentación) son relevantes, se esperaría que la obesidad, entre otras causas, refleje en mayor medida el impacto de los determinantes sociales en la salud de las ciudades de Oaxaca (Rivera, Hernández, Aguilar y Murayama, 2015). Los resultados del análisis bayesiano muestran que, dependiendo de la región, existe más de un efecto con mayor probabilidad en el territorio oaxaqueño. Por ejemplo, la desnutrición destaca en el Istmo y en la región del Papaloapan; el envejecimiento, en la Costa; la muerte infantil, en el Istmo y los Valles Centrales; y una mayor mortalidad, en la región de la Sierra Norte (Gráfica 2). No todas las causas predominan en todas las regiones de Oaxaca: en la región de los Valles Centrales y el Istmo destacan cuatro causas (desnutrición, envejecimiento, muerte infantil y mortalidad general), mientras que en el resto de las regiones solamente dos (Gráfica 4).

Por poseer la menor jerarquía en infraestructura y servicios en el sector salud, se esperaría que las ciudades que manifiestan los mayores impactos de los determinantes sociales en la salud de sus habitantes sean las pequeñas, como Ixtlán, Cuicatlán o Teotitlán. Sin embargo, visualizadas en conjunto y considerando la mortalidad por edad y municipio de residencia, las ciudades que resienten los mayores efectos en la salud de sus habitantes son las grandes y medianas. En tercer lugar, las zonas metropolitanas. El estado de salud determinado por los factores socioeconómicos se ve afectado en me- 
nor medida en las pequeñas ciudades (Gráfica 3). Específicamente, los resultados muestran que la urbe que manifiesta más impacto en la salud de sus habitantes es Puerto Escondido, una ciudad mediana; continúan Juchitán, Huatulco, Tlaxiaco y Huajuapan, grandes y medianas ciudades, antes que las zonas metropolitanas (la Zona Metropolitana de Tehuantepec está en el lugar nueve, y la Zona Metropolitana de Oaxaca en el trece). Las ciudades con menos impacto son Pinotepa, Matías Romero y Cuicatlán, las cuales son mediana y pequeña, respectivamente (Gráfica 5).

\section{Gráfica 4}

Regiones de Oaxaca: total de determinantes socioeconómicos en la salud

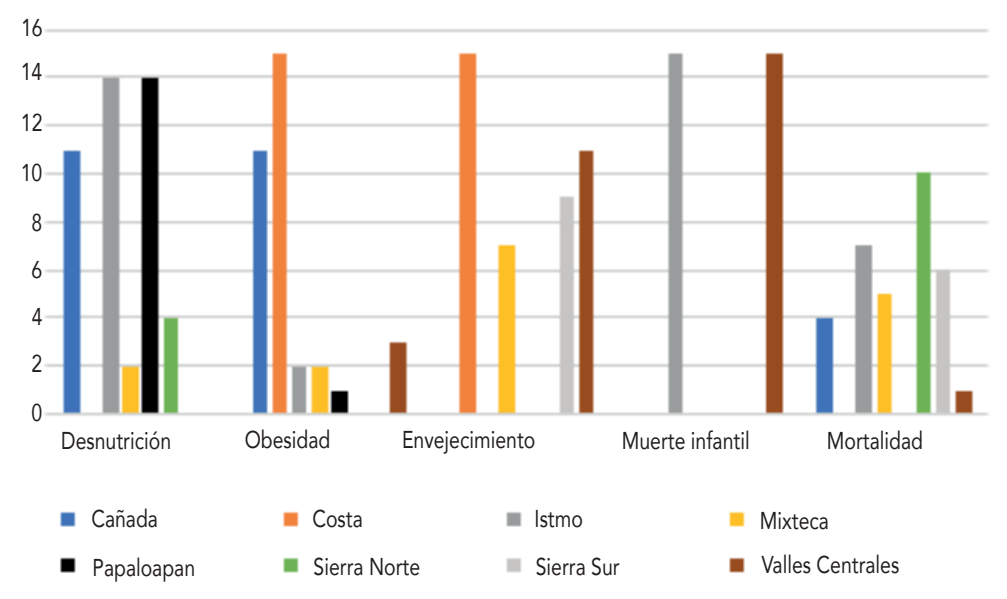

\footnotetext{
Fuente: Elaboración propia con base en el Cuadro 7.
}

En este contexto, también se esperaría que el envejecimiento, la muerte infantil, la desnutrición y la obesidad destaquen como los efectos más probables, sobre todo en las pequeñas ciudades de Oaxaca, por ser las que poseen la menor infraestructura y servicios de salud. Los resultados muestran que el envejecimiento destaca en las ciudades medianas; la desnutrición en las grandes ciudades; la obesi- 
dad en las pequeñas, y la muerte infantil en las zonas metropolitanas. En resumen, se observa que el predominio de los efectos en la salud de los factores socioeconómicos no posee un patrón homogéneo en el contexto de las ciudades analizadas (Gráfica 6).

\section{Gráfica 5}

Impacto de los factores socioeconómicos en las ciudades

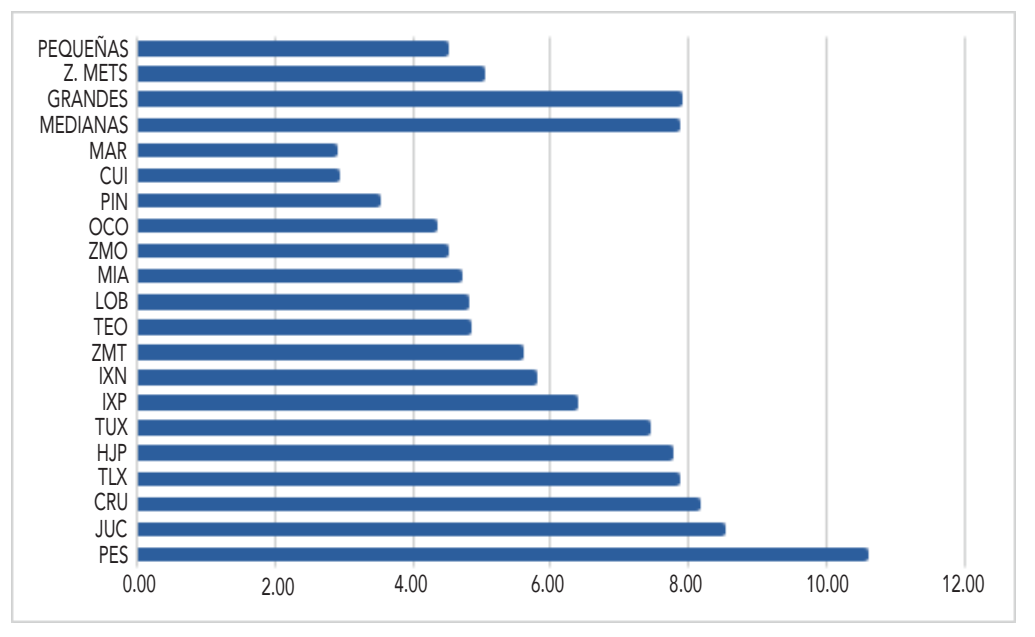

Nota: IXN: Ixtlán; CUI: Cuicatlán; TEO: Teotitlán; IXP: Ixtepec; LOB: Loma Bonita; MAR: Matías Romero; MIA: Miahuatlán; OCO: Ocotlán; PES: Puerto Escondido; TLX: Tlaxiaco; CRU: Huatulco; PIN: Pinotepa Nacional; HJP: Huajuapan; JUC: Juchitán; TUX: Tuxtepec; ZMT: Zona Metropolitana de Tehuantepec; ZMO: Zona Metropolitana de Oaxaca.

Fuente: Elaboración propia a partir de los Cuadros 4 y 5.

Dado el contexto socioterritorial del estado de Oaxaca, caracterizado por poseer indicadores bajos de bienestar en el contexto nacional, se esperaría que la pobreza y la desigualdad sean los determinantes socioeconómicos predominantes en la salud de la población de la mayoría de sus ciudades. Sin embargo, los resultados arrojan que la funcionalidad urbana (FEST) destaca en las ciudades de tamaño medio en Oaxaca; en segundo lugar, la educación. Otros determinantes que destacan, derivados del contexto socioterritorial, 
son las amenazas como el cambio climático, la no sustentabilidad y los conflictos sociales existentes en las ciudades. El bienestar, la gestión, la vivienda y la pobreza se ubican en el siguiente nivel. El resto de los determinantes socioeconómicos poseen la menor jerarquía según el contexto de las urbes analizadas (Gráfica 7).

\section{Gráfica 6}

Tipos de ciudades de Oaxaca:

factores de salud predominantes por factor socioeconómico

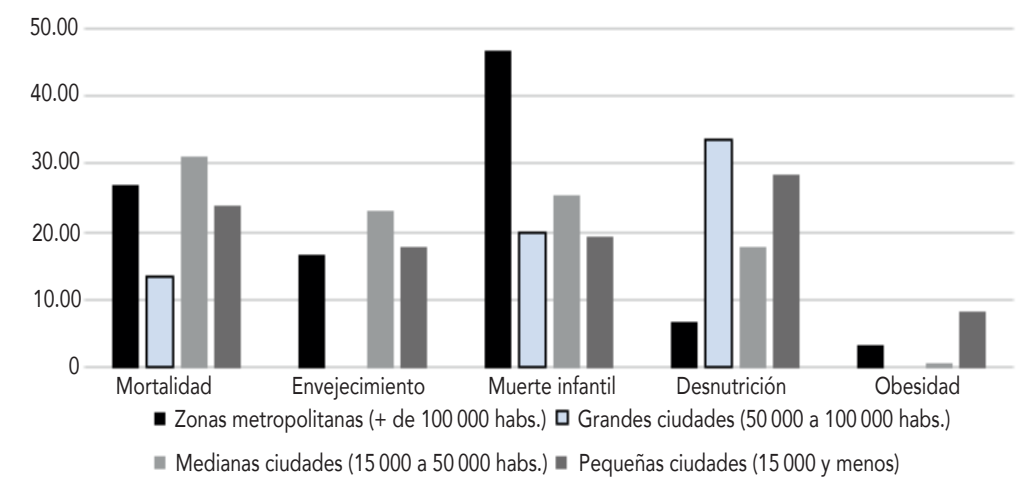

Fuente: Elaboración propia a partir de los Cuadros 4 y 5.

La red de los determinantes de salud y sus efectos, la cual posee un bajo nivel de interacciones (el coeficiente de Pearson respectivo es de 0.37 ), muestra que el agrupamiento más fuerte está compuesto por seis factores (ambiente, gestión, pobreza, fortalezas, oportunidades y amenazas); y los más débiles por dos (vivienda-educación y economía-funcionalidad urbana). La densidad de la red (0.32) es baja, lo que indica el relativamente poco entrelazamiento de los determinantes sociales analizados. La mayoría de los determinantes muestran un grado de centralidad igual a 1.0. La centralidad más baja (0.80) la manifiestan los determinantes economía y FEST, indicando que son los factores más débiles de la red (Cuadro 8). De igual manera, el grado de centralización de la red (0.038) puede considerarse muy bajo, indicando que no existe un determinante que ocupe el lugar central de la red y domine la dinámica del conjunto (Gráfica 8). 


\section{Gráfica 7}

Ciudades de Oaxaca: factores

socioeconómicos predominantes en la salud

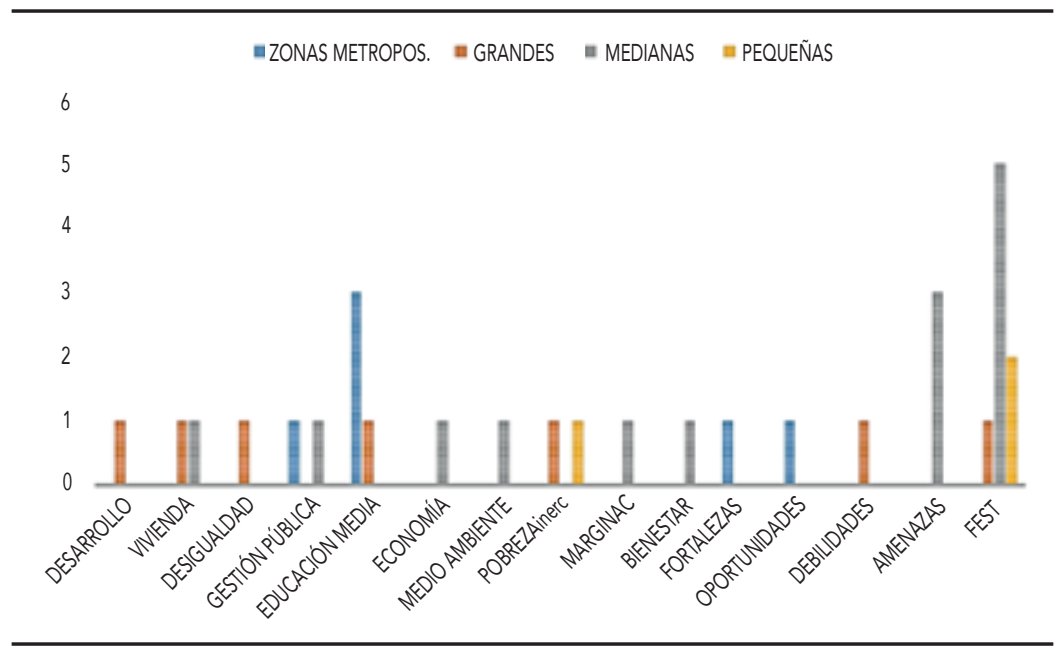

Nota: ZONAS METROPS.: zonas metropolitanas; POBREZAinerc: pobreza inercial; MARGINAC: marginación, FEST: funcionalidad urbana.

Fuente: Elaboración propia con base en los Cuadros 4 y 5.

A través de la red también se observa que la desnutrición está ligada a la educación y a la economía (en color más oscuro en la Gráfica 8). El envejecimiento preferentemente se enlaza con la desigualdad, las debilidades, las amenazas, el desarrollo y, en menor medida, con la FEST. La mortalidad se vincula con las oportunidades, la gestión pública y la pobreza. La muerte infantil, con las debilidades, la desigualdad, el desarrollo y el medio ambiente. Y la obesidad, con la educación, la economía, las oportunidades y la FEST.

\section{Conclusiones y discusión}

Con respecto a la interrogante del presente artículo acerca de si la estructura territorial -que se propone se manifiesta a través de la funcionalidad de las ciudades- puede considerarse un factor que genera inequidad sanitaria en el estado de salud de las mismas, la respuesta 
es afirmativa, pero de manera parcial. El análisis bayesiano mostró que la funcionalidad urbana (FEST) resultó el determinante socioeconómico más frecuente en algunas ciudades de Oaxaca, específicamente en Tlaxiaco, Puerto Escondido y Huajuapan, y su influencia se percibe mejor en las medianas y las grandes ciudades. A su vez, el análisis de redes confirmó que el peso de la organización espacial es relativamente débil todavía en las ciudades analizadas.

\section{Gráfica 8}

Red de los determinantes sociales de la salud en Oaxaca

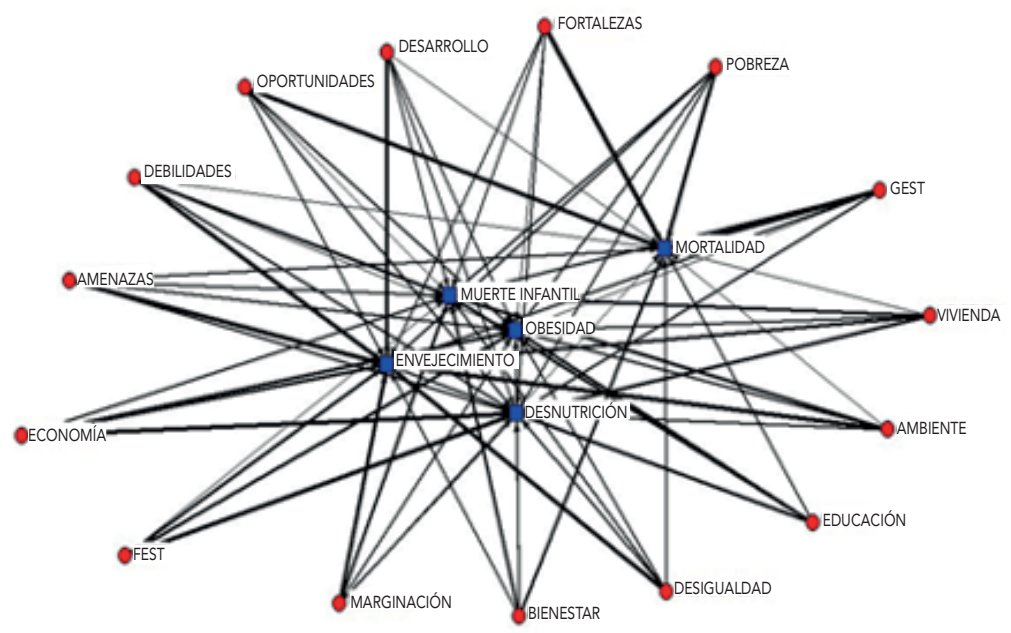

OPORTUNIDADES

Fuente: Elaborado con datos de los Cuadros 4 y 5 con el programa UCINET 6.6.

La aplicación del análisis bayesiano mostró que no existe un determinante social predominante para todas las ciudades, sino que dependiendo del nivel de desarrollo socioeconómico de cada ciudad, destaca(n) algún(os) determinante(s), pero en general, indica que la problemática de la salud relacionada con la territorialidad en este estado preferentemente está ligada a la pobreza, los bajos niveles de bienestar y la marginación, es decir, con la carencia de infraestructura y servicios básicos, como comunicaciones, agua potable, drenaje, etc. En gran medida, esto refleja la falta de inversiones en las ciu- 
dades, ligadas a los factores que pueden mejorar su funcionalidad, especialmente en las microrregiones habitadas por los pueblos originarios de Oaxaca.

La aplicación del teorema de Bayes matizó la problemática de la salud urbana en las ciudades oaxaqueñas, lo que puede contribuir a facilitar diagnósticos orientados a la toma de decisiones para optimizar los recursos públicos, ya que, de acuerdo con Barbini, Manzi y Barbini:

[...] aunque el enfoque bayesiano no es la única forma de abordar la problemática en la toma de decisiones bajo una incertidumbre, es sin duda una herramienta muy útil para ser utilizada en medicina y salud porque permite tomar decisiones con bases racionales, haciendo más fácil explicar y defender las decisiones elegidas [Barbini, Manzi y Barbini, 2013].

La interpretación del comportamiento de la red de factores socioeconómicos y de salud también confirma que son los determinantes socioeconómicos los que aún predominan en la salud de la población del territorio analizado. Así, por ejemplo, las interacciones de los factores sugieren que la desnutrición está ligada a la carencia de educación y al bajo ingreso, que depende de la economía. El envejecimiento, con la desigualdad, las debilidades, las amenazas, la falta de desarrollo y una débil funcionalidad urbana. La mortalidad, con la falta oportunidades, la deficiente gestión pública y la pobreza. La muerte infantil, con las debilidades, la desigualdad, el bajo nivel de desarrollo y el ambiente contaminado. La obesidad se vincula con la carencia de educación, el bajo ingreso proveniente de la economía, la débil funcionalidad y la falta de oportunidades en las ciudades.

A esto último se aúna la compleja geografía de Oaxaca, así como su atraso estructural en inversiones públicas, que justifica que la débil funcionalidad de la mayoría de sus ciudades aún esté ligada a la carencia de infraestructura y procesos que faciliten la movilidad social intra e interurbana. Cabe reflexionar que en las últimas décadas, el proceso de urbanización de las sociedades pone en evidencia la necesidad de cuidar las ciudades para que sus espacios ofrezcan una buena calidad de vida, lo cual incluye como condición básica la mo- 
vilidad intra e interurbana de personas y mercancías, pues la distribución desigual del acceso a los medios de transporte se transforma en una participación desigual del derecho de uso de la ciudad, así como de los beneficios y costos de la movilidad colectiva. Las personas de más bajos ingresos, así como las poblaciones rurales y originarias, sufren mucho más que el resto de las personas las consecuencias indeseables del uso del transporte individual, convirtiéndose esta situación en un grave problema para los países (y regiones) en desarrollo (Vasconcellos, 2010, p. 192; Venter, Mahendra e Hidalgo, 2019), especialmente para el mejoramiento de sus servicios de salud.

Esto refleja que la mayoría de las localidades oaxaqueñas se encuentran suburbanizadas y requieren de un mejoramiento en la infraestructura en la atención médica, ya que la seguridad social depende del equipamiento, instalaciones y servicios de diversas instituciones para la atención de sus afiliados. La falta de atención a esta problemática tiende a incrementar la vulnerabilidad en las zonas urbanas y en sus microrregiones de influencia, caracterizadas por la falta de acceso a los servicios de salud, educación y oportunidad laboral, factores predisponentes que, junto con una transición demográfica y epidemiológica, aumentan el predominio de enfermedades no transmisibles que en su gran mayoría son prevenibles (principalmente en los grupos vulnerables como los pueblos originarios de Oaxaca ) e incrementan la mortalidad.

Las ciudades de Oaxaca aún no satisfacen las necesidades de salud de sus habitantes, especialmente de la población originaria, ya que carecen de la infraestructura y el equipamiento necesarios para resolver la problemática respectiva; además, su población no siempre cuenta con el empleo ni el ingreso capaces de sustituir los apoyos públicos por los privados en caso de enfermedad. Todo ello ocasiona una amenaza para la salud de los ciudadanos (por los problemas de contaminación ambiental, la falta de planeación de su desarrollo, la inseguridad, la débil funcionalidad de las instituciones responsables, etc.). Esto puede mejorarse si se fortalece el sector salud con políticas públicas con un "enfoque intercultural", sobre todo en las ciudades con fuerte presencia de pueblos originarios, para contar con un elemento catalizador que facilite establecer puentes entre los servicios de salud y las diferentes culturas que conforman la población 
usuaria. En resumen, es menester adecuar los servicios a las características culturales de la población, promover los programas, y contar con mecanismos para la traducción en lenguas originarias en la impartición de los servicios (Almaguer, Vargas y García, 2014, p. 104).

En este contexto, por localizarse las ciudades analizadas en microrregiones de pueblos originarios, las conclusiones del estudio de Torres et al. (2003) siguen siendo válidas, pues los datos analizados confirman que en este contexto los grupos originarios enfrentan una doble carga de enfermedad: primero, por el rezago epidemiológico que sufren, en donde las muertes del grupo I de enfermedades transmisibles continúa siendo el patrón dominante; segundo, porque afrontan causas de defunción cuya tendencia al aumento es preocupante, pues esas comunidades tienen que hacerle frente con escasos recursos. Dada la débil funcionalidad urbana existente, una mejor infraestructura, equipamiento y apoyo a las ciudades en cuanto a la movilidad inter e intraurbana, al personal médico y administrativo en el área de la salud de las ciudades de Oaxaca y en las microrregiones bajo su influencia, podría reducir dicha carga, pues éstas pueden conformar redes de lugares centrales equipados, capaces de revertir o controlar territorialmente las enfermedades derivadas de la urbanización. 


\section{Cuadro 4}

Determinantes de salud y probabilidad bayesiana de las ciudades de Oaxaca

4a) Probabilidad bayesiana: desarrollo, vivienda, medio ambiente, bienestar, desigualdad, economía y educación

\begin{tabular}{|c|c|c|c|c|c|c|c|}
\hline$R E G$ & DSUS & $V I V$ & $A M B$ & BIEN & DESG & ECON & EDUC \\
\hline \multicolumn{8}{|l|}{ CAÑ } \\
\hline TEO & 7.5 & 8.1 & 4.3 & 7.1 & 6.9 & 30.8 & 4.73 \\
\hline CUI & 6.9 & 8.1 & 9.4 & 8.9 & 6.6 & 13.1 & 4.58 \\
\hline \multicolumn{8}{|c|}{ COST } \\
\hline PES & 10.8 & 10.4 & 13.4 & 11.9 & 10.5 & 68.7 & 8.47 \\
\hline CRU & 13.0 & 12.3 & 12.8 & 14.0 & 13.0 & 7.7 & 8.83 \\
\hline PIN & 8.3 & 9.5 & 7.8 & 10.7 & 8.0 & -0.1 & 7.9 \\
\hline \multicolumn{8}{|l|}{ IST } \\
\hline ZMT & 11.4 & 9.7 & 11.2 & 7.8 & 8.2 & 6.7 & 14.1 \\
\hline JUC & 13.7 & 12.7 & 12.8 & 12.7 & 15.2 & 33.1 & 10.34 \\
\hline IXP & 8.7 & 7.5 & 10.3 & 6.4 & 8.0 & 22.2 & 5.93 \\
\hline MAR & 4.8 & 8.1 & 6.2 & 10.5 & 7.7 & -0.1 & 6.54 \\
\hline \multicolumn{8}{|l|}{ MIX } \\
\hline HJP & 7.5 & 6.9 & 8.1 & 9.4 & 7.1 & 30.7 & 7.46 \\
\hline TLX & 7.6 & 8.3 & 10.7 & 2.4 & 7.2 & 75.4 & 6.42 \\
\hline \multicolumn{8}{|l|}{ PAP } \\
\hline TUX & 10.0 & 10.7 & 10.1 & 10.6 & 13.3 & -1.5 & 11.98 \\
\hline \multicolumn{8}{|l|}{ SN } \\
\hline IXN & 6.4 & 6.5 & 6.9 & 8.5 & 5.8 & 31.4 & 4.43 \\
\hline \multicolumn{8}{|l|}{ SS } \\
\hline MIA & 6.5 & 6.7 & 7.9 & 13.3 & 6.2 & 30.7 & 5.17 \\
\hline \multicolumn{8}{|l|}{ VC } \\
\hline ZMO & 9.8 & 8.4 & 8.3 & 8.4 & 9.7 & 0.0 & 28.6 \\
\hline $\mathrm{OCO}$ & 8.0 & 8.2 & 5.5 & 8.9 & 7.5 & 3.1 & 5.84 \\
\hline
\end{tabular}

Nota: DSUS: desarrollo; VIV: vivienda; AMB: medio ambiente; BIEN: bienestar; DESG: desigualdad; ECON: economía; EDUC: educación; REG: región; CAÑ: Cañada; COST: Costa; IST: Istmo; MIX: Mixteca; PAP: Papaloapan; SN: Sierra Norte; SS: Sierra Sur; VC: Valles Centrales. 
4b) Probabilidad bayesiana: gestión pública, marginación, pobreza, fortalezas, oportunidades, debilidades, amenazas y funcionalidad

\begin{tabular}{|c|c|c|c|c|c|c|c|c|}
\hline$R E G$ & GEST & $M A R G$ & POBRE & FORT & OPOR & $D E B I$ & AMEN & FEST \\
\hline \multicolumn{9}{|l|}{ CAÑ } \\
\hline TEO & 4.7 & 8.2 & 4.0 & 4.7 & 4.0 & 8.2 & 0.7 & 30.8 \\
\hline CUI & 4.6 & 9.4 & 3.5 & 4.6 & 3.5 & 9.4 & 0.2 & 13.1 \\
\hline \multicolumn{9}{|c|}{ COST } \\
\hline PES & 8.5 & 10.2 & 15.4 & 8.5 & 15.4 & 10.2 & 15.2 & 68.7 \\
\hline CRU & 8.8 & 12.9 & 12.7 & 8.8 & 12.7 & 12.9 & 8.9 & 7.7 \\
\hline PIN & 7.9 & 7.6 & 4.2 & 7.9 & 4.2 & 7.6 & 0.9 & -0.1 \\
\hline \multicolumn{9}{|l|}{ IST } \\
\hline ZMT & 14.1 & 9.0 & 11.0 & 14.1 & 11.0 & 9.0 & 12.5 & 6.7 \\
\hline JUC & 10.3 & 13.0 & 9.4 & 10.3 & 9.4 & 13.0 & 20.9 & 33.1 \\
\hline IXP & 5.9 & 8.1 & 4.3 & 5.9 & 4.3 & 8.1 & 25.6 & 22.2 \\
\hline MAR & 6.5 & 6.3 & 4.4 & 6.5 & 4.4 & 6.3 & 0.5 & -0.1 \\
\hline \multicolumn{9}{|l|}{ MIX } \\
\hline HJP & 7.5 & 7.4 & 7.8 & 7.5 & 7.8 & 7.4 & 8.1 & 30.7 \\
\hline TLX & 6.4 & 5.9 & 7.4 & 6.4 & 7.4 & 5.9 & 8.9 & 75.4 \\
\hline \multicolumn{9}{|l|}{ PAP } \\
\hline TUX & 12.0 & 10.5 & 9.7 & 12.0 & 9.7 & 10.5 & 7.1 & -1.5 \\
\hline \multicolumn{9}{|l|}{ SN } \\
\hline IXN & 4.4 & 7.5 & 5.2 & 4.4 & 5.2 & 7.5 & 11.7 & 31.4 \\
\hline \multicolumn{9}{|l|}{ SS } \\
\hline MIA & 5.2 & 7.9 & 4.7 & 5.2 & 4.7 & 7.9 & 3.2 & 30.7 \\
\hline \multicolumn{9}{|l|}{$\mathrm{VC}$} \\
\hline ZMO & 28.7 & 9.0 & 29.7 & 28.7 & 29.7 & 9.0 & 12.0 & 0.0 \\
\hline $\mathrm{OCO}$ & 5.8 & 7.9 & 5.7 & 5.8 & 5.7 & 7.9 & 8.8 & 3.1 \\
\hline
\end{tabular}

Nota: GEST: gestión pública; MARG: marginación; POBRE: pobreza; FORT: fortalezas; OPOR: oportunidades; DEBI: debilidades; AMEN: amenazas; FEST: funcionalidad.

Fuente: Elaboración propia con base en los indicadores de la serie censal de INEGI $(1990 ; 2000 ; 2010)$, en consulta interactiva Mortalidad, conjunto de datos de INEGI (1990 a 2017) y en la Encuesta Nacional de Salud y Nutrición, 2006. 


\section{Cuadro 5}

Factores de salud predominantes según la probabilidad bayesiana en las ciudades de Oaxaca

5a) Factores de salud según probabilidad bayesiana:

desarrollo, vivienda, medio ambiente, bienestar, desigualdad, economía y educación

\begin{tabular}{|c|c|c|c|c|c|c|c|}
\hline$R E G$ & DSUS & VIV & $A M B$ & BIEN & $D E S G$ & $E C O N$ & $E D U C$ \\
\hline \multicolumn{8}{|l|}{ CAÑ } \\
\hline TEO & OBE & OBE & OBE & OBE & OBE & OBE & OBE \\
\hline CUI & NUT & NUT & NUT & NUT & NUT & NUT & NUT \\
\hline \multicolumn{8}{|c|}{ COST } \\
\hline PES & ENV & ENV & ENV & ENV & ENV & ENV & ENV \\
\hline CRU & ENV & ENV & ENV & ENV & ENV & ENV & ENV \\
\hline PIN & OBE & OBE & OBE & OBE & OBE & OBE & OBE \\
\hline \multicolumn{8}{|l|}{ IST } \\
\hline ZMT & INF & INF & INF & INF & INF & INF & INF \\
\hline JUC & NUT & NUT & INF & INF & NUT & NUT & NUT \\
\hline IXP & INF & INF & INF & INF & INF & OBE & MOR \\
\hline MAR & INF & INF & INF & MOR & MOR & NUT & MOR \\
\hline \multicolumn{8}{|l|}{ MIX } \\
\hline НJP & ENV & MOR & OBE & MOR & ENV & OBE & ENV \\
\hline TLX & ENV & ENV & ENV & MOR & ENV & NUT & ENV \\
\hline \multicolumn{8}{|l|}{ PAP } \\
\hline TUX & NUT & NUT & NUT & NUT & NUT & NUT & $\mathrm{OBE}$ \\
\hline \multicolumn{8}{|l|}{ SN } \\
\hline IXN & MOR & NUT & MOR & NUT & MOR & NUT & MOR \\
\hline \multicolumn{8}{|l|}{ SS } \\
\hline MIA & ENV & ENV & ENV & MOR & ENV & ENV & ENV \\
\hline \multicolumn{8}{|l|}{$\mathrm{VC}$} \\
\hline ZMO & ENV & ENV & ENV & MOR & ENV & ENV & ENV \\
\hline $\mathrm{OCO}$ & INF & INF & INF & INF & INF & INF & INF \\
\hline
\end{tabular}

Nota: DSUS: desarrollo; VIV: vivienda; AMB: medio ambiente; BIEN: bienestar; DESG: desigualdad; ECON: economía; EDUC: educación; OBE: obesidad; MOR: mortalidad; ENV: envejecimiento; NUT: desnutrición; INF: muerte infantil; REG: región; CAÑ: Cañada; COST: Costa; IST: Istmo; MIX: Mixteca; PAP: Papaloapan; SN: Sierra Norte; SS: Sierra Sur; VC: Valles Centrales. 
5b) Factores de salud según probabilidad bayesiana: gestión pública, marginación, pobreza, fortalezas, oportunidades, debilidades, amenazas y funcionalidad

\begin{tabular}{|c|c|c|c|c|c|c|c|c|}
\hline$R E G$ & GEST & $M A R G$ & POBRE & $F O R T$ & OPOR & $D E B I$ & $A M E N$ & FEST \\
\hline \multicolumn{9}{|l|}{ CAN } \\
\hline TEO & MOR & OBE & MOR & MOR & MOR & OBE & OBE & OBE \\
\hline CUI & MOR & NUT & OBE & MOR & OBE & NUT & NUT & NUT \\
\hline \multicolumn{9}{|c|}{ COST } \\
\hline PES & ENV & ENV & ENV & ENV & ENV & ENV & ENV & ENV \\
\hline CRU & ENV & ENV & ENV & ENV & ENV & ENV & ENV & ENV \\
\hline PIN & OBE & OBE & OBE & OBE & OBE & OBE & OBE & OBE \\
\hline \multicolumn{9}{|l|}{ IST } \\
\hline ZMT & INF & INF & INF & INF & INF & INF & INF & INF \\
\hline JUC & NUT & NUT & NUT & NUT & NUT & NUT & NUT & NUT \\
\hline IXP & MOR & INF & MOR & MOR & MOR & INF & MOR & OBE \\
\hline MAR & MOR & INF & MOR & MOR & MOR & INF & MOR & NUT \\
\hline \multicolumn{9}{|l|}{ MIX } \\
\hline HJP & MOR & ENV & MOR & MOR & MOR & ENV & ENV & OBE \\
\hline TLX & MOR & ENV & MOR & MOR & MOR & ENV & ENV & NUT \\
\hline \multicolumn{9}{|l|}{ PAP } \\
\hline TUX & NUT & NUT & NUT & NUT & NUT & NUT & OBE & NUT \\
\hline \multicolumn{9}{|l|}{ SN } \\
\hline IXN & MOR & MOR & MOR & MOR & MOR & MOR & MOR & NUT \\
\hline \multicolumn{9}{|l|}{ SS } \\
\hline MIA & MOR & ENV & MOR & MOR & MOR & ENV & ENV & ENV \\
\hline \multicolumn{9}{|l|}{$\mathrm{VC}$} \\
\hline ZMO & ENV & ENV & OBE & ENV & OBE & ENV & ENV & ENV \\
\hline $\mathrm{OCO}$ & INF & INF & INF & INF & INF & INF & INF & INF \\
\hline
\end{tabular}

Nota: GEST: gestión pública; MARG: marginación; POBRE: pobreza; FORT: fortalezas; OPOR: oportunidades; DEBI: debilidades; AMEN: amenazas; FEST: funcionalidad; OBE: obesidad; MOR: mortalidad; ENV: envejecimiento; NUT: desnutrición; INF: muerte infantil.

Fuente: Elaboración propia con base en los indicadores de la serie censal de INEGI $(1990 ; 2000 ; 2010)$, en consulta interactiva Mortalidad, conjunto de datos de INEGI (1990 a 2017) y en la Encuesta Nacional de Salud y Nutrición, 2006. 


\section{Cuadro 6}

Frecuencia de los determinantes sociales y factores de salud

\begin{tabular}{lccccc}
\hline & \multicolumn{5}{c}{ Factores de salud } \\
\cline { 2 - 6 } Deter. sociales & Desnutrición & Obesidad & Envejecimiento & $\begin{array}{c}\text { Muerte } \\
\text { infantil }\end{array}$ & Mortalidad \\
\hline Desarrollo & 3 & 2 & 6 & 3 & 1 \\
Vivienda & 4 & 2 & 5 & 4 & 1 \\
Ambiente & 2 & 3 & 6 & 4 & 1 \\
Bienestar & 3 & 2 & 2 & 4 & 5 \\
Desigualdad & 3 & 2 & 6 & 3 & 2 \\
Economía & 6 & 4 & 4 & 2 & 0 \\
Educación & 4 & 4 & 2 & 4 & 3 \\
Gestión & 2 & 1 & 3 & 2 & 8 \\
Marginación & 3 & 2 & 6 & 4 & 1 \\
Pobreza & 2 & 3 & 2 & 2 & 7 \\
Fortalezas & 2 & 1 & 3 & 2 & 8 \\
Oportunidades & 2 & 3 & 2 & 1 & 7 \\
Debilidades & 3 & 1 & 6 & 4 & 1 \\
Amenazas & 2 & 3 & 6 & 2 & 3 \\
Funcionalidad & 6 & 4 & 4 & 1 & 0 \\
\hline
\end{tabular}

Fuente: Elaborado con datos de los Cuadros 4 y 5. 


\section{Cuadro 7}

Centralidad de los determinantes sociales

y de salud en las ciudades de Oaxaca

7a) Centralidad de determinantes sociales

\begin{tabular}{lccccc}
\hline Deter. sociales & Grado & 2-Local & Autovector & Proximidad & Intermediación \\
\hline Desarrollo & 1.000 & 0.973 & 0.264 & 1.000 & 0.006 \\
Vivienda & 1.000 & 0.973 & 0.264 & 1.000 & 0.006 \\
Ambiente & 1.000 & 0.973 & 0.264 & 1.000 & 0.006 \\
Bienestar & 1.000 & 0.973 & 0.264 & 1.000 & 0.006 \\
Desigualdad & 1.000 & 0.973 & 0.264 & 1.000 & 0.006 \\
Economía & 0.800 & 0.800 & 0.216 & 0.943 & 0.003 \\
Educación & 1.000 & 0.973 & 0.264 & 1.000 & 0.006 \\
Gestión & 1.000 & 0.973 & 0.264 & 1.000 & 0.006 \\
Marginación & 1.000 & 0.973 & 0.264 & 1.000 & 0.006 \\
Pobreza & 1.000 & 0.973 & 0.264 & 1.000 & 0.006 \\
Fortalezas & 1.000 & 0.973 & 0.264 & 1.000 & 0.006 \\
Oportunidades & 1.000 & 0.973 & 0.264 & 1.000 & 0.006 \\
Debilidades & 1.000 & 0.973 & 0.264 & 1.000 & 0.006 \\
Amenazas & 1.000 & 0.973 & 0.264 & 1.000 & 0.006 \\
Funcionalidad & 0.800 & 0.800 & 0.216 & 0.943 & 0.003 \\
urbana (FEST) & & & & & \\
\hline
\end{tabular}

7b) Centralidad de determinantes de salud

\begin{tabular}{lccccc} 
Deter. salud & Grado & 2-Local & Autovector & Proximidad & Intermediación \\
\hline Desnutrición & 1.000 & 1.000 & 0.457 & 1.000 & 0.137 \\
Obesidad & 1.000 & 1.000 & 0.457 & 1.000 & 0.137 \\
Envejecimiento & 1.000 & 1.000 & 0.457 & 1.000 & 0.137 \\
Muerte infantil & 1.000 & 1.000 & 0.457 & 1.000 & 0.137 \\
Mortalidad & 0.867 & 0.751 & 0.406 & 0.852 & 0.093 \\
\hline
\end{tabular}

Fuente: Elaboración propia con el programa UCINET 6.6, con datos de los Cuadros 4 y 5 . 


\section{Cuadro 8}

Agrupamiento de los determinantes sociales de la salud de las ciudades de Oaxaca

\begin{tabular}{lcl}
\hline Agrup. & Frecuencia & \multicolumn{1}{c}{ Integrantes } \\
\hline 1 & 6 & $\begin{array}{l}\text { Ambiente, gestión, pobreza, fortalezas, oportunidades, } \\
\text { amenazas }\end{array}$ \\
2 & 5 & $\begin{array}{l}\text { Desarrollo, bienestar, desigualdad, marginación, } \\
\text { debilidades } \\
\text { Vivienda, educación } \\
3\end{array}$ \\
4 & 2 & Economía, funcionalidad \\
\hline
\end{tabular}

Coef. phi de Pearson: 0.370

Densidad red: 0.3243 (bajo)

Grado centralización: 0.038 (muy bajo)

Fuente: Elaborado con el programa UCINET 6.6, con datos de los Cuadros 4 y 5.

\section{Bibliografía}

Almaguer, J., Vargas, V. y García, H. (2014). La interculturalidad como política de salud. En J. A. Almaguer, V. Vargas Vite, H. J. García Ramírez (coords.), Interculturalidad en salud. Experiencias y aportes para el fortalecimiento de los servicios de salud (pp. 99-134). Ciudad de México: Secretaría de Salud.

Barbini, E., Manzi, P. y Barbini, P. (2013). Bayesian approach in medicine and health management. En A. J. Rodríguez-Morales (ed.), Current topics in public health (pp. 17-35). Rijeka, Croacia: IntechOpen.

CDI. (Comisión Nacional para el Desarrollo de los Pueblos Indígenas). (2006). Regiones originarias de México. Programa de las Naciones Unidas para el Desarrollo, México. http://www.cdi. gob.mx/regiones/regiones_indigenas_cdi.pdf

CIEDD. (Centro de Información Estadística y Documental para el Desarrollo). (2014). Sistema de Información Municipal. Censos económicos, Oaxaca. Gobierno del Estado de Oaxaca. México. http://www.sim.oaxaca.gob.mx/ 
DGIS. (Dirección General de Información en Salud). (2000-2016). Cubos dinámicos. Ciudad de México. http://www.dgis.salud. gob.mx/contenidos/basesdedatos/BD_Cubos_gobmx.html

Fernández, J. (2006). Planificación estratégica de ciudades: nuevos instrumentos y procesos. Barcelona: Reverté.

Galea, S. y Vlahov, D. (2005). Urban health: Evidence, challenges and directions. Annual Review of Public Health, 26(1), 341-365. https://www.ncbi.nlm.nih.gov/pubmed/15760293

Gómez, F. J. (1999). Análisis geográfico, estructuras territoriales y sistemas nodales. En J. Vilá i Valentí (coord.), El seu mestratge en la geografia universitária (pp. 363-370). Barcelona: Universitat de Barcelona.

Guerra, C., Vila, J., Apolinaire, J., Cabrera, A., Santana, I. y Almaguer, P. (2009). Factores de riesgo asociados a sobrepeso y obesidad en adolescentes. Medi Sur. Revista Electrónica de las Ciencias Médicas en Cienfuegos, 7(2), 25-34. http://scielo.sld. $\mathrm{cu} / \mathrm{pdf} / \mathrm{ms} / \mathrm{v} 7 \mathrm{n} 2 / \mathrm{v} 7 \mathrm{n} 2 \mathrm{a} 632 . \mathrm{pdf}$

Hernández, L. (2016). Análisis de la centralidad y el desarrollo sustentable en la microrregión zapoteca Sierra Sur. (Tesis de maestría en Desarrollo Regional y Tecnológico, Instituto Tecnológico de Oaxaca, Tecnológico Nacional de México).

INEGI. (Instituto Nacional de Estadística y Geografía). (2000). Censo General de Población y Vivienda 2000. Principales resultados por localidad. Sistema de Integración Territorial, ITER 2000. Aguascalientes, México. http://www.inegi.org.mx/sistemas/consulta resultados/iter 2000.aspx? $\mathrm{c}=27437 \&$ s $=$ est

INEGI. (Instituto Nacional de Estadística y Geografía). (2005). Conteo de Población y Vivienda 2005. Principales resultados por localidad. Sistema de Integración Territorial, ITER 2005. Aguascalientes, México. https://www.inegi.org.mx/programas/ ccpv/2005/

INEGI. (Instituto Nacional de Estadística y Geografía). (2010). Censo de Población y Vivienda 2010. Tabulados básicos. Población total por municipio, sexo y grupos quinquenales de edad según tamaño de localidad. Aguascalientes, México. http://www3. inegi.org.mx/sistemas/TabuladosBasicos/Default.aspx?c=27302 
INEGI. (Instituto Nacional de Estadística y Geografía) (INEGI). (2010a). Cuéntame. Diversidad, Aguascalientes, México. http:// www.cuentame.inegi.org.mx/monografias/informacion/oax/ poblacion/diversidad.aspx

INEGI. (Instituto Nacional de Estadística y Geografía). (INEGI). (2013). Conociendo Oaxaca. Aguascalientes, México. http:// internet.contenidos.inegi.org. $\mathrm{mx} / \mathrm{contenidos} / \mathrm{productos} / \mathrm{prod}$ serv/contenidos/espanol/bvinegi/productos/estudios/conociendo/ OAXACA.pdf

INEGI. (Instituto Nacional de Estadística y Geografía). (INEGI). (2013a). Serie histórica censal e intercensal, 1990-2010. Aguascalientes, México. https://www.inegi.org.mx/programas/ccpv/ cpvsh/

INEGI. (Instituto Nacional de Estadística y Geografía). (INEGI). (2015). Marco geoestadístico nacional. Productos y servicios. Localidades. Aguascalientes, México. http://www.inegi.org.mx/ geo/contenidos/geoestadistica/consulta_localidades.aspx

INEGI. (Instituto Nacional de Estadística y Geografía). (INEGI). (2016). Estadísticas a propósito del Día Internacional de los Pueblos Originarios. Datos nacionales. Aguascalientes, México. https://www.contenidos-pearson.com/PDF\%20Historia\%202 /Historia_020.pdf

Instituto Nacional de Salud Pública. (2006). Encuesta Nacional de Salud y Nutrición 2006, 2012. Resultados por entidad federativa. Cuernavaca, Morelos. https://ensanut.insp.mx/encuestas/ ensanut2016/descargas.php

Kanuri, C., Revi, A., Espey, J. y Kuhle, H. (2019). Cómo implementar los ODS en las ciudades. Un manual introductorio para quienes trabajan en el ámbito del desarrollo urbano sostenible. Global Iniciative for the United Nations / German Cooperation. https://reds-sdsn.es/wp-content/uploads/2018/12/SDG-CitiesGuide-Spanish_edited_Final-version.pdf

López, O. (2004). La sustentabilidad urbana. Bitácora Urbano Territorial, 1(8), 8-14. https://revistas.unal.edu.co/index.php/bitacora/ article/view/18750/19645

Martínez, K. (2018). Análisis de la relación de la vivienda con TIC y el desarrollo sustentable de las ciudades de Oaxaca durante 
2000-2015. (Proyecto de tesis doctoral en Desarrollo Regional y Tecnológico, México, Instituto Tecnológico de Oaxaca, Tecnológico Nacional de México).

Mercado, Y. y Robles, J. (2016). Una aplicación de la regla de Bayes en ciencias de la salud. Ponencia presentada en el XXVI Simposio Internacional de Estadística. Sincelejo, Sucre, Colombia, 8 a 12 de agosto. http://gfnun.unal.edu.co/fileadmin/content/ eventos/simposioestadistica/documentos/memorias/Memorias 2016/Posters/49._Bayes_Ciencias_de_la_salud_Mercado Robles.pdf

Miguel, A., Torres, J., Hernández, L. y Moncada, M. (2016). El desarrollo sustentable y la centralidad de las localidades en una microrregión de pueblos originarios. El caso de la Sierra Sur de Oaxaca, México. Revista Desarrollo Local Sostenible, 9(25). http://www.eumed.net/rev/delos/25/centralidad.html

OMS. (Organización Mundial de la Salud). (2016). Global Report on Urban Health: Equitable Healthier Cities for Sustainable Development. http://www.who.int/iris/handle/10665/204715

ONU-Hábitat. (2011). Cities and climate change: Global report on human settlements 2011. Londres. http://www.zaragoza.es/ contenidos/medioambiente/onu/538-eng-ed2011.pdf

ONU-Hábitat. (2016). Urbanización y desarrollo: futuros emergentes. Ciudad de México. https://centrourbano.com/2016/11/11/ urbanizacion-desarrollo-futuros-emergentes-onu/

OPS. (Organización Panamericana de la Salud). (2008). Primera reunión del Foro Regional de Salud Urbana de la Organización Panamericana de la Salud. Hacia un marco conceptual de salud urbana yagendaparalaacciónen las Américas. CiudaddeMéxico.https:// www.paho.org/mex/index.php?option=com_docman\&view =download\&alias=358-primera-reunion-del-foro-regionalde-salud-urbana-de-la-organizacion-panamericana-de-la-salud \&category_slug $=$ ops-oms-mexico\&Itemid $=493$

Ordóñez, M. (2000). El territorio del estado de Oaxaca: una revisión histórica. Investigaciones Geográficas, Boletín del Instituto de Geografia, 42, 67-86. http://www.investigacionesgeograficas. unam.mx/index.php/rig/article/viewFile/59115/52102 
OSMAN. (Observatorio de Salud y Medio Ambiente de Andalucía). (2016). Urbanismo, medio ambiente y salud, julio. España. http:/www.osman.es/project/urbanismo-medio-ambiente-ysalud/ (consulta: 28 de octubre de 2017).

PNUD. (Programa de las Naciones Unidas para el Desarrollo en México). (2015). Índice de desarrollo humano para las entidades federativas. Ciudad de México. https://www.undp.org/content/dam/ mexico/docs/Publicaciones/PublicacionesReduccionPobreza /InformesDesarrolloHumano/PNUD_boletinIDH.pdf

Propin, E. y Sánchez-Crispín, Á. (2001). Características básicas de la estructura territorial de la economía mexicana. Investigaciones Geográficas, Boletín del Instituto de Geografia, 46, 148-163. http://www.scielo.org.mx/scielo.php?script=sci_arttext\&pid $=\mathrm{S} 0188-46112001000300011$

Reyes, H., Gómez, H., Torres, L., Tomé, P., Galván, G., González, M. y Gutiérrez, G. (2009). Necesidades de salud en áreas urbanas marginadas de México. Revista Panamericana de Salud Pública, 25(4), 328-336. https://www.scielosp.org/article/rpsp/2009. v25n4/328-336/

Rivera, J., Hernández, M., Aguilar, C., Vadillo, F. y Murayama, C. (2015). Obesidad en México. Recomendaciones para una politica de Estado. Ciudad de México: Universidad Nacional Autónoma de México.

Rodríguez, L. E. (2003). Obesidad: fisiología, etiopatogenia y fisiopatología. Revista Cubana de Endocrinología, 14(2). http:// scielo.sld.cu/scielo.php?script=sci_arttext\&pid=S1561-29 532003000200006

Salinas, J. (2019). Teorema de Bayes. España. https://www.ugr.es/ j salinas/bayes.htm

Salingaros, N. (2005). Teoría de la red urbana. En N. Salingaros, Principles of urban structure. Design, Science Planning (pp. 15-38). Amsterdam: Techne Press. http://zeta.math.utsa.edu/ yxk833/ urbanweb-spanish.pdf

Secretaría de Salud (2018). Cubos dinámicos. Ciudad de México. www.dgis.salud.gob.mx/contenidos/basesdedatos/BD_Cubos gobmx.html 
Sectur. (Secretaría de Turismo). (2013). Primer informe de labores 2012-2013. Ciudad de México. http://www.sectur.gob.mx/pdf/ InformesL/sectur_1er_informe_labores_2013.pdf

Semarnat. (Secretaría de Medio Ambiente y Recursos Naturales). (2015). Informe del Medio Ambiente. México. https://apps1. semarnat.gob.mx:8443/dgeia/informe15/tema/pdf/Informe15 completo.pdf

Serrano, J. (2000). Redes urbanas y sistema de ciudades de Alemania. Algunas precisiones de cara al siglo XXI. Papeles de Geografía, 31, 115-135. https://revistas.um.es/geografia/article/view/47461

Sevitra. (Secretaría de Vialidad y Transporte). (2017). Reordenar, fortalecer y desarrollar el transporte es una prioridad. Oaxaca. https://www.oaxaca.gob.mx/comunicacion/reordenar-fortalecer -y-desarrollar-al-transporte-es-prioridad-sevitra/

Torres, J., Villoro, R., Ramírez, T., Zurita, B., Hernández, P., Lozano, R. y Franco, F. (2003). La salud de la población originaria en México. Caleidoscopio de la salud. Ciudad de México: Fundación Mexicana para la Salud.

Vasconcellos, E. (2010). Análisis de la movilidad urbana. Espacio, medio ambiente y equidad. Bogotá: CAF.

Venter, C., Mahendra, A. e Hidalgo, D. (2019). From mobility to access for all: Expanding urban transportation choices in the global south. (Documento de trabajo). Washington, DC: World Resources Institute. https://wrirosscities.org/sites/default/files/ WRR_Transport.pdf

Villegas, $\bar{C}$. (2016). De las ciudades multiculturales a las ciudades de inconformes. Algunas reflexiones sobre la protesta urbana en el siglo veintiuno. Bifurcaciones. Revista de Estudios Culturales Urbanos, 21. http://www.bifurcaciones.cl/2016/09/de-las-ciudades -multiculturales-a-las-ciudades-de-inconformes/

\section{Acerca de los autores}

Andrés Enrique Miguel Velasco es doctor en Ciencias en Planificación de Empresas y Desarrollo Regional por el Instituto Tecnológico de Oaxaca, México. Actualmente es profesor- investigador de la Di- 
visión de Estudios de Posgrado e Investigación en el Instituto Tecnológico de Oaxaca. Su línea de investigación es el desarrollo regional sustentable. ORCID: https://orcid.org/0000-0003-3319-0499

Entre sus publicaciones se encuentran:

Pérez, M., Miguel, A., Moreno, J. y Martínez, K. (2019). Educación media superior y desarrollo sustentable en las ciudades del estado de Oaxaca. Perfiles Educativos, 41(163), 70-87. http:// www.scielo.org.mx/pdf/peredu/v41n163/0185-2698-peredu -41-163-69.pdf

López, M., Miguel, A., Lambarria, G. y Martínez, K. (2018). Cambio climático y desarrollo de las ciudades de Oaxaca. México: Universidad Autónoma Benito Juárez de Oaxaca / Instituto Tecnológico de Oaxaca

Hernández, B., Miguel, A., López, M., Pérez, M., Moncada, M., Moreno, J. y Martínez, K. (2018). Desarrollo urbano y turismo en las ciudades emergentes de los valles centrales de Oaxaca, México. Oaxaca: Universidad Autónoma Benito Juárez de Oaxaca / Instituto Tecnológico de Oaxaca.

Andrés Miguel Cruz es médico cirujano por la Universidad Popular Autónoma del Estado de Puebla, titulado con mención honorífica. Realizó el internado rotatorio en el Hospital General "Dr. Manuel Gea González" y el servicio social en investigación en el Departamento de Gastroenterología del Instituto Nacional de Ciencias Médicas y Nutrición "Salvador Zubirán”. Es maestro en Salud Pública por la Universidad Popular Autónoma del Estado de Puebla. Sus líneas de investigación son: salud urbana y farmacovigilancia. ORCID: http://orcid.org/0000-0002-2258-2705

Entre sus publicaciones se encuentra:

Rivadeneyra, L., Miguel, A., Robles, C., Soto, E., Ceja, L. y González, M. (2017). Efectos de las bebidas con cafeína en la calidad de sueño de los alumnos de medicina en Puebla. CIMEL, 22(2), 30-34. http://oaji.net/articles/2017/6297-1531096141.pdf

Karina Aidee Martínez García es maestra en Ciencias en Desarrollo Regional y estudiante del Doctorado en Ciencias en Desarrollo Regional y Tecnológico en el Instituto Tecnológico de Oaxaca, Mé- 
xico. Su línea de investigación actual es el desarrollo regional sustentable. ORCID: http://orcid.org/0000-0002-8481-0902

Entre sus publicaciones se encuentran:

Pérez, M., Miguel, A., Moreno, J. y Martínez, K. (2019). Educación media superior y desarrollo sustentable en las ciudades del estado de Oaxaca. Perfiles Educativos, 41(163), 70-87. http:// www.scielo.org.mx/pdf/peredu/v41n163/0185-2698-peredu -41-163-69.pdf

Martínez, K. (coord.). (2018). La vivienda en el desarrollo sustentable de las pequeñas, medianas y grandes ciudades de Oaxaca. México: Universidad Autónoma Benito Juárez de Oaxaca / Instituto Tecnológico de Oaxaca.

López, M., Miguel, A., Lambarria, G. y Martínez, K. (2018). Cambio climático y desarrollo de las ciudades de Oaxaca. México: Universidad Autónoma Benito Juárez de Oaxaca / Instituto Tecnológico de Oaxaca.

Luz Astrid Martínez Sánchez es maestra en Derecho Fiscal y Administración Tributaria por la Universidad Anáhuac. Actualmente es estudiante del Doctorado en Ciencias en Desarrollo Regional y Tecnológico por el Instituto Tecnológico de Oaxaca, México. Su línea de investigación actual es el desarrollo regional sustentable. ORCID: http://orcid.org/0000-0003-4047-9311

Lizbeth Fabiola García Cruz es licenciada en Informática por el Instituto Tecnológico de Oaxaca, México. Actualmente es estudiante de la Maestría en Ciencias en Desarrollo Regional y Tecnológico en el Instituto Tecnológico de Oaxaca, México. Su línea de investigación actual es el desarrollo regional sustentable. La tesis que elabora para obtener el grado de maestra se titula "Análisis del empleo en el desarrollo regional sustentable de las ciudades de Oaxaca durante el periodo 2000 a 2017". ORCID: http://orcid.org/0000-0003-32591186

Recepción: 11 de abril de 2019. Aceptación: 10 de septiembre de 2019. 
